PHYSICS REPORTS (Review Section of Physics Letters) 237, No. 6 (1994) 325-355.

North-Holland

\title{
Necessary conditions for microscopic many-body theories *
}

\author{
A.D. Jackson and T. Wettig ${ }^{1}$ \\ Department of Physics, State University of New York, Stony Brook, New York, NY 11794, USA
}

Received August 1993; editor: G.E. Brown

Contents:

1. Introduction 327

2. Necessary conditions for saturation $\quad 328$

3. A simple theorem $\quad 330$

4. Random phase approximation for Bose fluids 334

5. Random phase approximation for Fermi fluids $\quad 337$

6. Beyond the random phase approximation 339
7. Parquet theory 342

8. Failures of parquet theory 349

9. Summary and conclusions 351

Appendix A. Approximate fermion RPA 352

Appendix B. Some two-potential results 353

References $\quad 355$

Abstract:

Familiar necessary conditions for the saturation of many-body binding energies are reconsidered in order to provide a better understanding of the analyticity properties of the ground state energy and of the convergence properties of certain sums of Goldstone diagrams. Using these results and other elementary arguments, we identify a minimum class of diagrams which must be summed to all orders if the perturbative treatment of other diagrams is to converge.

* Work supported in part by the US Department of Energy under grant No. DE-FG02-88ER 40388.

${ }^{1}$ E-mail address: wettig@nuclear.physics.sunysb.edu 


\title{
NECESSARY CONDITIONS FOR MICROSCOPIC MANY-BODY THEORIES
}

\author{
A.D. JACKSON and T. WETTIG
}

Department of Physics, State University of New York, Stony Brook, New York, NY 11794, USA

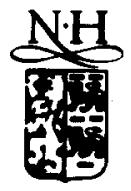

NORTH-HOLLAND 


\section{Introduction}

The problem of constructing reliable microscopic descriptions of strongly interacting quantum fluids has attracted interest and attention for many years and in many areas of physics. Solutions to this problem are of evident physical importance for systems as diverse as nuclear matter, the Coulomb gas, liquid helium and hard-sphere systems. In spite of this interest, much work remains to be done. The promise in the notion of using the nucleus as a "laboratory" in which one can probe other interactions remains unrealized due to present inabilities in solving the microscopic many-body problem. In the present paper, we shall be concerned with diagrammatic approaches to the many-body problem. (We shall not address the closely related questions of "variational" calculations of many-body systems to any significant degree.) The generic strategy in crafting an approach to the many-body problem is to identify a class (or classes) of diagrams which either should be or, more often, can be summed to all orders. Once this class is established, one (i) establishes an algorithm to sum the desired diagrams given a suitable driving term and (ii) seeks a systematic rearrangement of the full set of diagrams in order to construct this driving term (which, in most schemes, reduces to the bare interaction in the lowest order of implementation). In practice, one usually imagines that this driving term can be improved perturbatively. Most often, technical difficulties thwart implementation of this improvement beyond some low order. In principle, however, one should prove the sufficiency of any scheme proposed by actually demonstrating the convergence of any perturbative expansion of this driving term. This is a challenging task which is generally not attempted. A far simpler task is to demonstrate the necessity of summing a given class of diagrams. That is the aim of the present paper. We shall offer rather simple arguments aimed at identifying a minimum class of diagrams which must be included (to all orders) in any satisfactory approach to the many-body problem. This minimum class is most easily described as the self-consistent sum of all particle-particle ladder diagrams and all particle-hole chain diagrams. Specifically, we shall show that any attempt to treat some subset of these diagrams by perturbation theory will necessarily fail.

In section 2 we shall review certain conditions on the dynamics adopted for a many-body system which are necessary to ensure that the energy of the system is bounded from below. In section 3 we shall see that these simple results lead directly to the fact that the ground-state energy of a many-body system (in three dimensions) cannot be an analytic function of the strength of the underlying two-body interaction in the limit as this strength goes to zero. A diagrammatic interpretation of these results will be offered in section 4, where we consider the random phase approximation for Bose systems, and in section 5, where we consider Fermi systems. Section 6 addresses the limits on the convergence of the particle-particle ladder diagrams (most familiar in the "Brueckner theory" approach to nuclear matter and neutron matter). This section also considers the importance of providing a simultaneous description of long wave length excitations (ordinary sound) and short-distance correlations between particles in the fluid. These features will be seen to be incompatible in model theories which fall short of the self-consistent summation of particleparticle and particle-hole ladder diagrams. This self-consistent set of diagrams, commonly known as the "parquet" diagrams, will be considered in section 7 where we will also discuss the dilute limit of a gas of hard-sphere bosons in some detail. The failures of the parquet diagrams are interesting and physically significant in their own right. We shall consider them in section 8 before proceeding 
to a brief conclusion in section 9 .

Our overall goal is to identify approaches to the many-body problem which are rich enough to work and practical enough to give real solutions to real problems. In this spirit, we will attempt to base our arguments on simple and uncontroversial physics and to avoid the rather heavy formalism which often characterizes this problem.

\section{Necessary conditions for saturation}

Some years ago Calogero and coworkers established a number of conditions necessary for the binding energy of any infinite, many-body system to saturate [1]. For our purposes, "saturation" means that the total binding energy of a system of particles grows linearly with the number of particles. Equivalently, the system will saturate if the energy per particle is bounded from below. Although restricted to local two-body potentials, this work led to a number of necessary conditions by exploiting the tensor structure possible in the nucleon-nucleon interaction. For simplicity, consider the case when the two-body interaction contains only local (spin- and isospin-dependent) central forces. The qualitative content of the argument is straightforward. If the volume is fixed, the onebody kinetic energy makes a positive contribution of order $A^{2 / 3}$ (or $\rho^{2 / 3}$ ) to the energy per particle. In the thermodynamic limit, $A \rightarrow \infty$, this contribution is overwhelmed by the contribution of the central potential which is of order $A$ (or $\rho$ ). As a consequence, the various necessary conditions obtained apply equally to Fermi and Bose systems. It is easiest to consider Bose systems and a trial wave function which has the simple product form

$$
\Psi=\prod_{i=1}^{A} \psi\left(r_{i}\right)
$$

Clearly, a necessary condition for saturation is that the coefficient of the term of order $A$ is non-negative. This immediately tells us that

$$
\int \mathrm{d}^{3} r \mathrm{~d}^{3} r^{\prime}|\psi(r)|^{2} V\left(r-r^{\prime}\right)\left|\psi\left(r^{\prime}\right)\right|^{2} \geq 0
$$

for every choice of $\psi(r)$. Specific necessary conditions emerge from specific choices of $\psi(r)$. For example, choosing $\psi(r)$ equal to a constant leads to the condition that

$$
\int \mathrm{d}^{3} r V(r) \geq 0
$$

This is clearly equivalent to the requirement that $V(k=0) \geq 0$, where $V(k)$ is the Fourier transform of the potential in question. One might be tempted to make a more general argument based on the fact that eq. (2) is a simple convolution integral which can be rewritten as

$$
\int \mathrm{d}^{3} k\left[|\psi|^{2}(k)\right]^{2} V(k) \geq 0
$$

Since $\psi$ is arbitrary, one might wish to conclude that $V(k)$ must be greater than 0 for all $k$. This would be correct if $|\psi(r)|^{2}$ were any function. Since it is merely any positive function, the conclusion does not follow. ${ }^{*}$

*) The error in this argument is made clear by considering any totally repulsive potential which falls off more sharply than exp $\left[-(r / b)^{2}\right]$. By an elementary variational argument, the energy of such a system is larger than the energy of the non-interacting system and must saturate. However, the Fourier transform of such a potential is not positive for all momenta. 
A realistic potential for the description of a self-bound system must contain both attraction (for the system to be bound) and repulsion (for the system to saturate). Equation (2) makes it clear that the attraction must have longer range than the repulsion. [Otherwise, we could give $\psi$ a range shorter than that associated with the repulsive part of the interaction and violate eq. (2).]

Some of the Calogero conditions are surprising and counter-intuitive. This is to be expected since he is probing two-body dynamics for many-body pathologies which inevitably led to the consideration of "unreasonable" and "unphysical" density distributions. Since the aim was to judge the suitability of existing models of two-body (e.g., nucleon-nucleon) interactions and since virtually all were measured and found wanting, this work tended to generate more anger than understanding. One example should suffice. A two-body interaction composed of local central forces and a local spin-orbit force cannot saturate independent of the details of these forces. In a sense this result is "obvious". As noted above, the central force will make a contribution of order $A$ to the binding energy per particle. A spin-orbit force can make a contribution of $A^{4 / 3}$. $^{*}$ Since the expectation value, $\langle L \cdot S\rangle$, does not have a well-defined sign, one can always arrange a suitable polarization of spin and orbital angular momenta so that the coefficient of the $A^{4 / 3}$ term will be negative. The system will not saturate. Bethe responded to this result by noting that Calogero's spin-orbit instability was of a global nature and thus not of particular concern. In this case, one would only discover this difficulty by exploiting high values of $L$ for which there is neither theoretical nor empirical evidence for a local spin-orbit interaction. Such global pathologies could always be cured through the introduction of suitable nonlocal interactions or additional forms (such as a quadratic spin-orbit interaction). According to this point of view, the only relevant question was the local stability of physically interesting solutions; a subject not addressed by Calogero's arguments. The tacit assumption was that physically "reasonable" interactions, when used in conjunction with microscopically reliable many-body theories, would yield a physically interesting local minimum in the energy of a many-body system.

While this "physical" response has strong appeal, it is not necessarily appropriate. It can certainly complicate the already difficult task of learning how to perform reliable many-body calculations. The absence of stable local energy minima and the presence of energy pathologies in any given calculation can reflect either a defect in the interaction or a defect in the many-body theory. One does not know which. Consider, for example, the hypernetted chain class of "variational" calculations for Bose systems $\left.{ }^{* *}\right)$ in the trivial case of no interaction. The energy of the system is clearly non-negative. However, due to the approximations made in relating the basic two-body correlations in the wave function to the two-body distribution function, $g(r)$, the HNC energy has no lower bound [4]. This is clearly a defect of the many-body theory. However, in the presence of a two-body potential which binds the system, it may be very difficult to apportion blame reliably between the interaction and the various many-body approximations. The many-body problem is sufficiently challenging that, given the choice, one should never work with interactions known to be pathological.

Let us consider a particularly simple variational calculation for an interaction which violates eq. (3) using a non-interacting wave function made up of plane waves with the density, $\rho$, as a variational parameter. The energy per particle in this case is simply

$$
E / A \sim \rho^{2 / 3}-a \rho .
$$

*) The additional factor of $A^{1 / 3}$ comes from the presence of the operator $L \cdot S$. In the limit of high densities one would expect that $\langle L \cdot S\rangle \sim k_{\mathrm{F}} \sim \rho^{1 / 3} \sim A^{1 / 3}$.

**) These calculations are based on a simple Jastrow product wave function and an approximate relation between the two-body correlation function and the two-body distribution function $[2,3]$. 
There are three interesting densities in this problem. The largest of these is simply $\rho=1 / a^{3}$. For all larger densities, $E / A$ is negative. For all densities larger than $8 / 27 a^{3}$, the derivative of the energy is negative. Starting our calculations at any such density, we will be led inexorably to an infinitely dense system with infinite binding energy. (For smaller densities, we will be led to the infimum at $\rho=0^{+}$.) The third interesting density is the density at which the compressibility of the system, defined as

$$
C=(\partial / \partial \rho)\left(\rho^{2} \partial E / \partial \rho\right)
$$

changes sign. This occurs at $\rho=125 / 729 a^{3}$. For smaller densities, the compressibility is positive and the system is stable with respect to long-range, infinitesimal fluctuations in the density. However, for larger densities, the compressibility is negative. Long-range fluctuations will blow up exponentially. This is an indicator of the physical observation that locally stable, homogeneous density distributions cannot exist. Evidently, this energy function has no local minima. Long wave length fluctuations will lead us smoothly to the pathology at high densities.

\section{A simple theorem}

We believe that there is far greater uncertainty in the construction of adequate approximate microscopic many-body theories than there is in our knowledge of two-body dynamics (for most systems). Thus, it is our aim to redirect the focus of Calogero's results away from dynamics and towards those necessary conditions which approximate many-body theories must satisfy. For example, it seems reasonable to require that a trustworthy many-body theory should be capable of recognizing pathologies in the dynamics when such pathologies exist. This is particularly true of the long wave length diseases noted in the preceding section.

Towards this end, let us first establish a simple theorem which will rule out the most widely used approach to the many-body problem - simple perturbation theory. We consider an infinite manybody system (of fermions or bosons) whose dynamics is governed by a local two-body interaction, $\lambda V(r)$. The theorem is elementary: The equilibrium ground-state energy of this system is not an analytic function of $\lambda$ as $\lambda \rightarrow 0$. $^{*}$ As a consequence, the description of the energy of this system by perturbation theory (starting from the non-interacting system) cannot converge for any $\lambda$ no matter how small.

The proof of this theorem is simpler than its statement. Consider the two potentials $+\lambda V(r)$ and $-\lambda V(r)$. One of them must violate eq. (3) and lead to a ground-state energy without lower bound. Since eq. (3) is a necessary but not sufficient condition, the energy of the system might be unbounded from below in both cases (hence, our footnote). This is physically uninteresting. In physically interesting cases, the energy for, e.g., $+\lambda V(r)$ will be bounded from below. The energy for $-\lambda V(r)$ will not. Evidently, the point $\lambda=0$ must represent the location of some non-analytic feature. $\left.{ }^{* *}\right)$

One can undoubtedly sharpen this theorem. We shall rather turn to the simple special case with $V(r)>0$ for all $r$ for which the ground state energy has a lower bound of zero. To prove this, use the true wave function obtained with the potential $+\lambda V(r)$ as a trial wave function for the noninteracting system. It will, of course, provide an upper bound on the energy of the non-interacting

\footnotetext{
*) To be more careful, one should state that either this theorem holds or the energy is unbounded from below for all $\lambda$ and, hence, the system is of no physical interest.

**) Usually, this will be a branch point or even an accumulation point for branch points as will be indicated below.
} 
ground state. Since $+\lambda V(r)$ is always positive, this variational estimate for the energy of the non-interacting system will always be lower than the exact energy for the system with potential $+\lambda V(r)$. The exact non-interacting ground-state energy is evidently 0 for bosons and positive for fermions. Hence, for such potentials, $+\lambda V(r)$ has a finite lower bound while $-\lambda V(r)$ has no lower bound.

This theorem certainly raises questions regarding the validity and significance of, for example, virial expansions. We do not wish to suggest that such approaches are without merit. Rather, it seems more likely that these expansions are asymptotic expansions which can be improved within limits. It remains an interesting question to. provide practical criteria for assessing their validity.

While we do not claim the above theorem as "new", we believe that it is nevertheless "news" for many. It is the many-body analogue of Dyson's familiar argument in quantum electrodynamics. Dyson argued that QED cannot be analytic in its coupling constant, $e$ [5]. His argument was to make the continuation $e^{2} \rightarrow-e^{2}$ so that the interaction between electrons (or between positrons) becomes attractive while the electron-positron interaction becomes repulsive. With this replacement, it is energetically favorable to create electron-positron pairs (at a cost which is linear in the number of pairs produced). The electrons will then clump together at high density (as will the positrons). The resulting energy will be attractive and proportional to the square of the number of pairs produced. The energy of the vacuum for $e^{2}<0$ will be unbounded from below while the energy for $e^{2}>0$ will have a lower bound. As above, perturbative calculations in $e^{2}$ will not converge.

Formal work by a number of authors has revealed similar divergences in the perturbative treatment of other model quantum field theories [6-8]. The general approach has been to investigate the asymptotic behaviour of high-order terms in the perturbation series, and the general result is a factorial growth of the expansion coefficients. It is possible to deal with these problems by identifying the series with its Borel summation. While this prescription is ad hoc, it is abundantly clear that simple-minded perturbation theory is not adequate.

The problems of QED can be related to the masslessness of the photon [9]. Calculations to finite order in $e^{2}$ can result in infrared singularities in certain Feynman diagrams. It is possible to eliminate these singularities by summing a related (infinite) class of diagrams which contain an arbitrary number of (soft) photon insertions. (In practise, this can be done using the Bloch-Nordsieck approximation [10] or eikonal techniques.) The analogy to many-body theory will become clear in section 4 where we will see that it is necessary to sum processes involving arbitrarily many (soft) particle-hole excitations in order to avoid infrared singularities in many-body Bose systems. (This is essentially the content of the random phase approximation.) In these cases, perturbation theory is not only divergent, but individual terms are not even well defined.

How should one then regard the enormous efforts invested in perturbative calculations for QED [11]? Given the fact that the various corrections calculated to date grow smaller with order, it seems likely that QED is an asymptotic series. In the absence of general results for arbitrary orders in $e^{2}$, we have no way of knowing when it is appropriate to terminate this series. Nevertheless, a finite number of terms can provide useful (although not conclusive) guidance. Consider the function

$$
S(x)=\int_{0}^{\infty} \mathrm{d} t \mathrm{e}^{-t}\left(\frac{1}{1+t / x}+\frac{1}{t / x} \ln (1+t / x)\right)
$$

and the related asymptotic series

$$
S_{N}=\sum_{n=0}^{N}(-1)^{n}\left(\frac{n+2}{n+1}\right) \frac{n !}{x^{n}},
$$


for the case $x=1000$. Imagine that we have the ability to calculate any individual term in this series but not the general term. ${ }^{*}$ Let us retain the first three terms in this sum and construct the absolute value of the ratios of successive terms, $r_{n}$. Fitting this limited information regarding $r_{n}$ with a linear function of $n$ indicates that $r_{n}$ equals 1 (and the series should be terminated) at $N=972$. This is in remarkable agreement with the correct answer. While this estimate is by no means rigorous, it is remarkably useful given the ease of its construction.

Another possible view of QED is apparently more high-powered. Looking down from QCD, one might claim that QED should not be regarded as a basic theory at all. Rather, it should be obtained by starting from the Standard Model and integrating out unwanted high-energy degrees of freedom. If this scheme were actually carried out, QED (as an effective theory) would emerge along with rules for its proper use. One might then assert that these rules would preclude operations exploiting Dyson's pathology. This stance would seem to be unacceptable. Just as in the many-body example above, Dyson's argument is governed by the infrared properties of QED. Using Earnshaw's theorem, one would expect (classically) that long wave length fluctuations would take us smoothly from the normal vacuum to Dyson's disaster when $e^{2}<0$. While it is completely normal for effective theories to have spurious ultraviolet problems, experience suggests that they should be well behaved in the infrared.

Of course, the arguments here and in the preceding section are dependent on the spatial dimension of the problem at hand. They depend on the fact that, for $d=3, E / A$ receives a contribution of order $\rho^{2 / 3}$ from the kinetic energy and a (dominant) contribution of order $\rho$ from the potential energy. For spatial dimension $d$, the kinetic term is of order $\rho^{2 / d}$. For $d \leq 2$, we are not entitled to assume the dominance of the potential energy. While the Calogero result, eq. (2), does not hold, one might still expect to find non-analytic behaviour of the energy as a function of the interaction strength. Such behaviour is familiar. Consider, for example, the half-filled Hubbard model in one dimension. In this model, spin 1/2 fermions reside on $N$ lattice sites. There are equal numbers of spin up and spin down fermions, and the total number of particles is equal to the total number of lattice sites. The kinetic energy operator is replaced by a finite difference form,

$$
\sum_{k \sigma}\left(a_{k+1 \sigma}^{\dagger} a_{k \sigma}+a_{k-1 \sigma}^{\dagger} a_{k \sigma}\right)
$$

There is an interaction of strength $U$ between any two fermions (of opposite spin) occupying the same lattice site. In the thermodynamic limit the ground-state energy is [12]

$$
E \sim \int_{0}^{\infty} \mathrm{d} \omega \frac{J_{0}(\omega) J_{1}(\omega)}{\omega(1+\exp 2 z \omega)}
$$

Here, $J_{0}$ and $J_{1}$ are cylindrical Bessel functions and $z=U / 8$. The integral is finite for all values of $U$ including the value $U=0$ for which this integral is equal to $1 / \pi$. Each derivative of this energy with respect to $U$ will bring in one additional power of $\omega$ under the integral. Evidently, the second (and each higher) derivative is not defined at $U=0$ and can only be obtained by some suitable limiting process $U \rightarrow 0^{ \pm}$. It is also elementary to see that

$$
E(-U)=2 / \pi-E(+U) \text {. }
$$

*) Evidently, the best we can do is to stop at $N=1000$ with the confidence that the exact result is bounded by $S_{1000}$ from above and $S_{1001}$ from below. But we do not know this! 
Continuity of $E$ and all its derivatives thus requires that all even derivatives of $E$ with respect to $U$ vanish at $U=0$. (Continuity sets no requirements on the odd derivatives.) In fact, the situation is maximally non-continuous. Using the limiting procedure, one finds that all odd derivatives (greater than the first) at $0^{+}$are zero while all even derivatives are nonzero. Specifically, using eq. (11), we see that all even derivatives of this energy are discontinuous at $U=0$. A naive power series expansion can be made (for $U \geq 0$ ) about the point $U=0^{+}$using these derivatives to obtain

$$
\begin{aligned}
& E(z)=1 / \pi-z / 4+\sum_{m=1} c_{m} z^{2 m}, \\
& c_{m}=\frac{(2 m-1)\left(2^{2 m+1}-1\right)[(2 m-3) ! !]^{3}}{2^{3 m-1}(m-1) !} \frac{\zeta(2 m+1)}{\pi^{2 m+1}} .
\end{aligned}
$$

Here, $\zeta(n)$ is the Riemann zeta function. This series diverges for all $U$ [13].

The divergence of this series is equivalent to the failure of perturbation theory starting from the non-interacting ground state. The easiest way to understand the origin of this problem was offered by Takahashi who wrote eq. (10) as a sum of hypergeometric functions [14]. This allowed him to show that $E(U)$ has infinitely many logarithmic branch points at $z= \pm \mathrm{i} / m$, where $m$ is any positive integer. The point $U=0$ becomes an accumulation point of branch points. With this analytic structure in mind, $U=0$ would appear to be the worst possible place to start a series expansion of $E(U)$.

A simple mathematical remedy exists for this mathematically expressed problem. Specifically, since all of the branch points are logarithmic, it is most convenient to let the cuts of $E(U)$ lie along the locus of branch points. Hence, $E(U)$ is cut from $z=+i$ to $z=-i$. Knowledge of the discontinuity of $E(U)$ would be helpful but is hard to get. Fortunately, knowledge of the location of the cut is sufficient to construct a perturbative representation of $E(U)$ which is maximally convergent (in a sense to be defined below). Since a perturbation expansion about $z_{0}$ will converge only inside the largest circle which does not touch the cut, the strategy is evident. We seek a conformal mapping, $z \rightarrow t$, such that the cut in $E(z)$ is mapped onto the unit circle in the complex $t$-plane. We further require that the entire first Riemann sheet (which includes the positive real $U$ axis) is mapped onto the interior of this unit circle. In the present case, the desired mapping is a textbook example:

$$
z=\frac{1}{2}(1 / t-t)
$$

There are infinitely many other mappings which could have been chosen. All will have the same radius of convergence in the mapped variable. (Any conformal mapping which does not take the cut onto the unit circle will necessarily bring some portion of the cut inside the unit circle. The radius of convergence for such mappings will be smaller.)

The strategy for obtaining the desired expansion, $E(t)$, is straightforward. Return to the defining integral and make the replacement

$$
1 /(1+\exp 2 z \omega) \rightarrow(\exp \omega t) /(\exp \omega / t+\exp \omega t)
$$

Expand the denominator as a geometric series recognizing that the first term, exp $\omega / t$, is dominant for all $t$ of interest. Then, expand the various factors of exp $n \omega t$ as a power series in $t$. The result of these operations is neither elegant nor transparent. Numerical studies verify the expectation that the resulting power series in $t(U)$ is convergent for all $U$ on the first Riemann sheet. The resulting 
form is not suitable for evaluating $E(-U)$. Physically, in going from $0^{+} \rightarrow 0^{-}$, we go through the cut and not around it. The energy for $U<0$ is not on the first Riemann sheet. It is easy to obtain $E(-U)$ by computing $E(+U)$ and then using eq. (11) - which is always valid - to determine $E(-U)$.

Several comments are in order. Other remedies exist for this problem. For example, the series defined by eqs. (12) and (13) is Borel resummable. Based on the arguments above, it seems likely that the non-analytic behaviour at $U=0$ exhibited by the Hubbard model is not merely an unfortunate artifact of an exactly soluable model but rather a reflection of more general analyticity problems associated with the use of perturbative descriptions of interacting many-body systems based on the non-interacting states. The "cure" we have affected through the use of a conformally mapped variable has a clear physical analogue in any "diagrammatic" many-body theory. We should identify those processes which lead to non-analytic behaviour of the energy (as a function of interaction strength) and resum them. Any additional processes included must respect this resummation. Failure to follow this procedure will result in a description of the many-body system which will not converge.

\section{Random phase approximation for Bose fluids}

In this section we shall consider the approximation to the static and dynamic structure functions and the ground-state energy of an infinite Bose system which results from summing all diagrams which are fully particle-hole reducible. $\left.{ }^{*}\right)$ This class of diagrams - the diagrams of the Random Phase Approximation (RPA) - will be seen to have the kind of analyticity structure required by the general theorem noted above. While this observation will not establish the "sufficiency" of performing such RPA summations, it will establish their "necessity".

We start with the dynamic structure function, $S(k, \omega)$, as shown diagrammatically in fig. 1 . We consider a local potential whose Fourier transform is $V(k)$. (Here and elsewhere in this paper we shall measure the potential and its Fourier transform in units of $\hbar^{2} / m$.) The RPA consists of summing a geometric series. The only technical difficulty lies in performing the integration of the product of two free Green's functions over the loop four-momentum.

We start with the free Green's function for a single particle,

$$
G_{0}^{\alpha \beta}(k, \omega)=\delta_{\alpha \beta}\left(\frac{\theta\left(k-k_{\mathrm{F}}\right)}{\omega-\omega_{k}+\mathrm{i} \eta}+\frac{\theta\left(k_{\mathrm{F}}-k\right)}{\omega-\omega_{k}-\mathrm{i} \eta}\right) .
$$

Here, it is economical (and legitimate) to describe the Bose system as a Fermi system with an artificial degeneracy, $\nu{ }^{* *)}$ We will also adopt the notation

$$
\omega_{k}=k^{2} / 2 \text {. }
$$

The Bose limit is obtained by letting $k_{\mathrm{F}} \rightarrow 0$ and $\nu \rightarrow \infty$ in such a fashion that the density (which is equal to $\nu k_{\mathrm{F}}^{3} / 6 \pi^{2}$ ) remains constant. The integral over the loop momentum (and the sum over the internal fictitious quantum number) is now simply done to obtain the bare polarization function,

$$
\Pi^{0}(p, \omega)=-\mathrm{i} \nu \int \frac{\mathrm{d}^{4} q}{(2 \pi)^{4}} G_{0}(q) G_{0}(q+p)=\frac{2 \rho \omega_{p}}{\left(\omega-\omega_{p}+\mathrm{i} \eta\right)\left(\omega+\omega_{p}-\mathrm{i} \eta\right)} .
$$

*) "Fully ph reducible" means that any given diagram of order $n$ in the interaction can be separated into two disjoint pieces by cutting one particle and one hole line. Further, each of the resulting pieces can be similarly reduced. This process of reduction can be continued until we are left with $n$ disconnected factors of the (bare) potential.

**) The indices $\alpha$ and $\beta$ label this fictitious quantum number. 
The dynamic liquid structure function in the RPA is written as

$$
S(p, \omega)=(\mathrm{i} / \rho) \Pi^{0}(p, \omega) /\left[1-V(p) \Pi^{0}(p, \omega)\right] .
$$

It is most revealing to recast the dynamic structure function as

$$
\begin{aligned}
& S(p, \omega)=\mathrm{i} 2 \omega_{p} /\left(\omega-\epsilon_{p}+\mathrm{i} \eta\right)\left(\omega+\epsilon_{p}-\mathrm{i} \eta\right), \\
& \epsilon_{p}=\sqrt{\omega_{p}^{2}+2 \rho \omega_{p} V(p)} .
\end{aligned}
$$

The dynamic structure function describes the response of the system to an external probe which transfers four-momentum $(p, \omega)$ to the system. Although interesting in its own right, we are more interested in the static structure function which is defined as

$$
S(p)=\int \frac{\mathrm{d} \omega}{2 \pi} S(p, \omega) .
$$

Given our explicit form for the dynamic structure function, we find

$$
S(p)=\omega_{p} / \epsilon_{p}=\omega_{p} / \sqrt{\omega_{p}^{2}+2 \rho \omega_{p} V(p)} .
$$

This result is of considerable interest. We first note the trivial (but correct) result that $S(p)=1$ for the non-interacting system. For interactions which meet the simplest necessary condition for saturation, $V(p=0)>0$, we see that the small-p behaviour of $S(p)$ is

$$
\lim _{p \rightarrow 0} S(p)=p / \sqrt{4 \rho V(0)}
$$

The static structure function vanishes linearly with $p$ in this limit with a coefficient which does not admit a convergent power series expansion in powers of the potential. Said more generally, the fact that $V(0)>0$ guarantees that an expansion of $S(p)$ in powers of $V(p) / \omega_{p}$ will not be

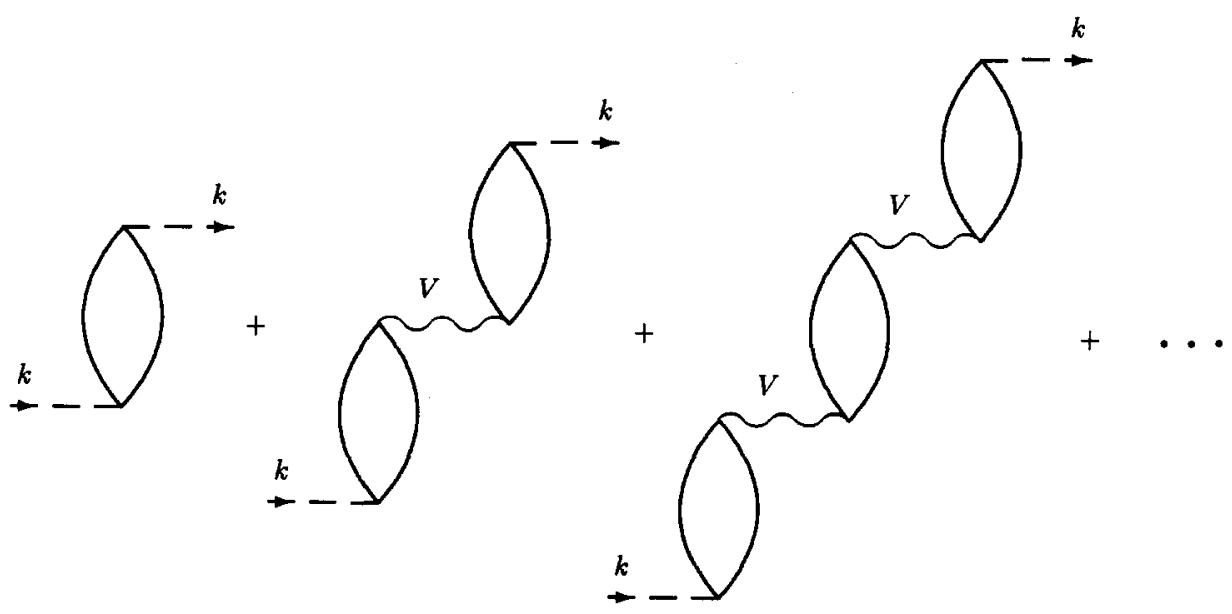

Fig. 1. The liquid structure function in the random phase approximation. 
uniformly convergent for all $p$. The physical content of this remark can be emphasized by recalling the Bijl-Feynman approximation for the energy, $\epsilon_{p}$, of a phonon of wave number $p[15,16]$,

$$
\epsilon_{p} \sim \omega_{p} / S(p) .
$$

In the non-interacting case, the phonon energy is strictly $p^{2} / 2$. In the interacting case, however, the phonon energy vanishes linearly with $p$ for sufficiently small $p$ independent of the strength of the interaction. ${ }^{*)}$ Since the constant of proportionality between $\epsilon_{p}$ and $p$ is simply the sound velocity, the RPA predicts that the velocity of sound does not have a convergent perturbative expansion. While these results are suggestive, our simple theorem addresses the analytic properties of the ground-state energy to which we now turn.

The ground-state energy is quickly obtained from the static structure function using the FeynmanHellmann theorem,

$$
\frac{E}{A}=\frac{1}{2} \rho V(0)+\frac{1}{2} \int_{0}^{1} \mathrm{~d} \lambda \int \frac{\mathrm{d}^{3} p}{(2 \pi)^{3}} V(p)\left[S_{\lambda}(p)-S_{0}(p)\right] .
$$

In this expression, $S_{\lambda}(p)$ is the static structure function obtained with the interaction $\lambda V$. The non-interacting structure function, $S_{0}(p)$, is simply 1 as we have noted. However, writing the Feynman-Hellmann theorem in this form permits immediate generalization to Fermi systems. We shall exploit this fact in the next section.

The coupling constant integration in this expression for the energy appears to prevent an $n$-fold overcounting of terms of order $V^{n}$. It is easily performed analytically,

$$
\frac{E}{A}=\frac{1}{2} \rho V(0)+\frac{1}{2} \int \frac{\mathrm{d}^{3} k}{(2 \pi)^{3}} V(k)\left(-1-\frac{k^{2}}{2 \rho V}+\frac{k^{2}}{2 \rho V} \sqrt{1+4 \rho V / k^{2}}\right) .
$$

It is useful to consider performing the integral in this expression by first performing an expansion of the square root. The first non-vanishing term is of order $V^{2}$. At this order, the integrand is nonsingular as $k \rightarrow 0$ and will vanish as $k \rightarrow \infty$ in a fashion determined by the nature of the potential. This second-order contribution to $E / A$ is finite. However, all higher-order terms are singular as $k \rightarrow 0$. As a consequence of the non-uniform convergence of the perturbative expansion of $S(k)$, we find that we cannot even construct a perturbative expansion for the energy in powers of the strength of the potential. Of course, we could imagine a complete recasting of an exact many-body theory in which some suitable effective interaction would drive the RPA diagrams. (This would result in some modification of counting factors as well.) Since we cannot preclude the possibility that the perturbative expansion of this effective ph interaction will also diverge, we cannot claim that summing the RPA is sufficient. The present exercise suggests, however, that exact summations of the RPA diagrams are necessary.

It is interesting to note that the integrand appearing in eq. (26) is negative for all values of $4 \rho V(k) / k^{2}>-1$. The RPA necessarily lowers the energy below the value provided by lowest-order perturbation theory.

It is evident that the RPA contains singularities related to those considered above. Specifically, if $V(0)<0$, it is clear that the square root appearing in the RPA energy will have some region of negative argument. The non-analyticity of the energy as a function of $\lambda$ in the vicinity of $\lambda=0$ is reflected in the RPA energy. To make this point clearer (and to reveal an inadequacy of the

*) The range in $p$ for which this linear approximation holds does depend on interaction strength. 
RPA), it is useful to consider a specific class of potentials - Gaussians, for example - such that both $V(r)$ and $V(k)$ are everywhere positive. ${ }^{*)}$ For the moment let us imagine that $\lambda$ is small and positive. We define

$$
k_{0}=\sqrt{4 \rho \lambda} .
$$

For sufficiently small $\lambda$ (or sufficiently small density), $k_{0}$ will be smaller than any momentum, $\mu$, characterizing the potential. It is now convenient to consider three contributions to the energy. The leading term is simply $\rho \lambda / 2$. For $k>k_{0}$ we can approximate the integrand in eq. (26) by the first non-vanishing term (of second order in $\lambda$ ). This term will make an attractive contribution of order $-\rho \lambda^{2} \mu$ to the energy. For $k<k_{0}$ we must retain the square root but are entitled to make the approximation that $V(k) \approx V(0)$. This leaves us with an elementary integral and an attractive contribution to the energy of order $-\rho^{3 / 2} \lambda^{5 / 2}$. This final term makes explicit the singularity at $\lambda=0$.

It is also interesting to consider the case of $\lambda$ fixed (and positive) in the limit $\rho \rightarrow 0$. We see that the first and second terms just considered are of the same dominant order (linear in $\rho$ ). These terms have different dependence on the interaction strength and are of opposite sign. For sufficiently large $\lambda$, the attractive term is dominant, and we find an RPA energy which is attractive and proportional to $\rho \lambda^{2}$. Since we are considering a purely repulsive interaction, this result is unphysical. It is a clear indication of the fact that, while RPA is necessary, it is not sufficient. We shall return to this point below.

\section{Random phase approximation for Fermi fluids}

The RPA for fermions is somewhat more complicated than the manipulations of the previous section. The origin of this complication can be seen in eq. (18) where we note that the denominator acquires a nontrivial dependence on the momentum $q$ when $k_{\mathrm{F}}$ is nonzero. The energy of a system of fermions in the RPA thus requires a two-dimensional integration rather than the one-dimensional integration of eq. (26) (see ref. [17] in this regard ). In order to make things clearer, we shall adopt an approximate treatment of the RPA for fermions. (This approximation is described in some detail in appendix A.) We emphasize, however, that we have verified the validity of our various qualitative conclusions based on this approximation by numerical studies.

The exact free static structure function is

$$
S_{0}(k)= \begin{cases}\frac{3}{4} k / k_{\mathrm{F}}-\frac{1}{16}\left(k / k_{\mathrm{F}}\right)^{3} & \text { if } k \leq 2 k_{\mathrm{F}}, \\ 1 & \text { if } k>2 k_{\mathrm{F}} .\end{cases}
$$

We note that this non-interacting fermion structure function vanishes linearly as $k \rightarrow 0$ while the non-interacting Bose structure function is 1 . This difference is likely to have significant effects on various convergence arguments. The Bijl-Feynman argument above suggested that the expected proportionality between the energy and momentum of a long wave length phonon could only be obtained in an interacting system. This is evidently not the case for fermions since $S_{0}(k) \sim k$ as $k \rightarrow 0$ even for non-interacting particles. The associated (approximate) structure function for interacting fermions is derived in appendix $A$,

$$
S(k)=S_{0}(k)\left[1+4 \rho V(k) S_{0}^{2}(k) / k^{2}\right]^{-1 / 2} .
$$

*) Since we are making the strength of the potential explicit, we set $V(k=0)=1$. 
Here, we shall assume two species of fermions so that

$$
\rho=\left(1 / 3 \pi^{2}\right) k_{\mathrm{F}}^{3} \text {. }
$$

The corresponding RPA energy for fermions can again be obtained from the Feynman-Hellmann theorem. Of course, the kinetic energy of the non-interacting fermions, absent in our previous boson example, must be added. The result is

$$
\begin{aligned}
& E / A=\frac{3}{5} \hbar^{2} k_{\mathrm{F}}^{2} / 2 m+\frac{1}{2} \rho V(0) \\
& +\frac{1}{2} \int \frac{\mathrm{d}^{3} k}{(2 \pi)^{3}}\left[\frac{k^{2}}{2 \rho S_{0}(k)}\left(1+4 \rho S_{0}^{2}(k) \frac{V(k)}{k^{2}}\right)^{1 / 2}-\frac{\underline{k^{2}}}{2 \rho S_{0}(k)}-V(k) S_{0}(k)\right] \text {. }
\end{aligned}
$$

The first term is always positive, the second term is positive for saturating potentials, and the third term is always negative.

This form raises two questions. First, can we construct a series expansion of this RPA energy and, if so, what is its radius of convergence? Second, does the Fermi RPA energy reflect Calogero's pathologies? The answer to the first question lies again in the argument of the square root. We note that $S_{0}^{2}(k) / k^{2}$ is a monotonically decreasing function of $k$ with the value $9 / 16 k_{\mathrm{F}}^{2}$ at $k=0$ and the value $1 / k^{2}$ for $k \geq 2 k_{\mathrm{F}}$. For smooth potentials [such that $V(k)$ is also monotonically decreasing], it is clear that the small- $k$ behaviour of this argument is of greatest interest. The vanishing of this argument sets the radius of convergence of a series expansion at

$$
|V(0)| k_{\mathrm{F}} \leq \frac{4}{3} \pi^{2}
$$

As repeatedly noted, this result has come from an approximate expression. A similar analysis of the exact RPA is considerably more tedious but merely yields a slightly different numerical prefactor,

$$
|V(0)| k_{\mathrm{F}} \leq \pi^{2} .
$$

This result is markedly different from the boson RPA for which the radius of convergence of the RPA diagrams is strictly 0 .

The situation for both bosons and fermions is perfectly consistent with the expected instabilities of the system when the volume integral of the potential is less than zero. Let us see how this works. For bosons the basic starting point is the elementary variational energy obtained from all particles in the $k=0$ state at a density $\rho$. As noted, the energy is

$$
E / A=\frac{1}{2} \rho V(0) .
$$

The compressibility, defined as $\partial_{\rho}\left(\rho^{2} \partial E / \partial \rho\right)$, is negative for all $V(0)<0$ indicating that the system is unstable with respect to long wave length fluctuations. These fluctuations are described by long wave length phonons. Since a small (but macroscopic) change in the density of the system can be thought of as a "condensate" of such $k=0$ phonons, the indicator of this instability must be that the sound velocity goes to (and through) zero. Returning to the Bijl-Feynman expression and using the RPA form for the static structure function, we see that we encounter the instability as $V(k=0) \rightarrow 0$. The RPA does not provide a sufficiently rich description of the system to actually describe the macroscopic density fluctuation. However, running into the square root branch point tells us precisely what the system wants to do. The location of this instability sets the radius of convergence of the RPA. This radius of convergence, which also governs the case $V(0)>0$, happens to be zero for bosons. 
Exactly the same situation applies to fermions. For fermions our starting point involves placing particles (with a degeneracy $\nu=2$ ) in the states $0 \leq k \leq k_{\mathrm{F}}$. The resulting variational energy at density $\rho$ differs from the boson result only by the addition of the kinetic energy term, $\frac{3}{5} \hbar^{2} k_{\mathrm{F}}^{2} / 2 \mathrm{~m}$. Writing the energy in terms of the density we find

$$
E / A=\frac{3}{10}\left(3 \pi^{2} \rho\right)^{2 / 3}+\frac{1}{2} \rho V(0) .
$$

The compressibility for this system has the form

$$
C=\frac{1}{3} k_{\mathrm{F}}^{2}+\left(1 / 3 \pi^{2}\right) k_{\mathrm{F}}^{3} V(0) \text {. }
$$

The condition that the system should be unstable with respect to long wave length fluctuations is simply

$$
k_{\mathrm{F}} V(0) \leq-\pi^{2}
$$

This is precisely the condition previously obtained from the exact RPA form of the fermion $S(k)$, leading to eq. (32). Again, Bijl-Feynman provides the interpretation that passing beyond this zero means that a long wave length phonon will lower the energy of the system. Again, encountering the square root branch point - either in $S(k)$ or $E / A$ - tells us that the system wants to undergo a macroscopic change in its density in order to exploit the Calogero pathology. Again, the location of this instability sets the radius of convergence of the RPA. For the fermion case, this radius of convergence is $|V(0)|<\pi^{2} / k_{\mathrm{F}}$. The stability condition that the compressibility of eq. (35) be positive corresponds to the familiar statement in Fermi Liquid Theory that $F_{0}$ must be greater than $-1[18]$.

\section{Beyond the random phase approximation}

Of course, the RPA is not sufficient to deal with the delicate balance of attraction and strong repulsion which characterizes "realistic" interactions for either nuclear matter or atomic liquids. It is evident that perturbation theory is not up to the task of summing the particle-particle ladder processes which build in the related short range correlations. Specifically, in the low-density limit, the sum of particle-particle ladder diagrams reduces essentially to the usual Lippman-Schwinger equation describing the scattering of two particles in free space. ${ }^{*}$ ) The radius of convergence for the perturbative sum of pp ladders is obtained by the replacement $V \rightarrow \lambda V$. One then considers a slightly unusual eigenvalue equation by looking for the spectrum, $\lambda_{n}$, such that $\lambda_{n} V$ has a bound state of energy $E_{0}$. If there exist any $\left|\lambda_{n}\right|<1$, a perturbative treatment of pp ladders will fail. If the potential in question actually has a weakly bound state (e.g., the deuteron in the ${ }^{3} S_{1}-{ }^{3} D_{1}$ channel of the NN system), there will certainly be an eigenvalue close to $\lambda=1$. This is rarely the eigenvalue with the smallest magnitude and does not set the radius in question. Given the necessity of strong short-range repulsion, the smallest eigenvalue will be negative corresponding to a potential with short-range attraction. For example, for the Reid ${ }^{1} \mathrm{~S}_{0} \mathrm{NN}$ potential and $E_{0}=0$, the smallest eigenvalues are +1.084 (which indicates the proximity of a two-body bound state) and -0.063 (which measures the strength of the short-range repulsion and establishes the desired radius of

*) This integral equation is usually modified slightly by the appearance of a Pauli operator, and is usually evaluated "off-shell" at some appropriate negative energy, $E_{0}$, on the order of $-20 \mathrm{MeV}$ for nuclear matter. These details are not particularly relevant. 
convergence). ${ }^{*)}$ Evidently, for potentials with very strong repulsion (such as the $1 / r^{12}$ behaviour of the Lennard-Jones potential) any attractive coefficient will lead to infinitely many bound states. Hence, in this case, the desired radius of convergence is strictly zero. In short, virtually all realistic interactions require that particle-particle ladder diagrams are summed to all orders.

To consider the consequences of this additional all-order summation, it is useful to consider the extreme example of a hard-core potential of radius $a$. Such potentials have been studied in many-body systems with considerable care over more than forty years (see, e.g., refs. [19-21]). The generic argument is roughly as follows. Every diagram which is pp reducible is ph irreducible. Thus, each of the terms in the full sum of pp ladder diagrams constitutes a satisfactory driving term for the RPA. If the density is relatively low or the range of the core is relatively small (i.e., if $\left.\rho a^{3} \ll 1\right)$, it is sensible to approximate the sum of all particle-particle ladder diagrams by the free-space two-body scattering amplitude at zero energy. Specifically, for a potential of height $V_{0}=\kappa^{2}$ and range $a$, this corresponds to the identification

$$
\bar{V}(p)=4 \pi a\left(\frac{\sin p a}{p a}-\cos p a \frac{\tanh \kappa a}{\kappa a}\right) \frac{\kappa^{2}}{p^{2}+\kappa^{2}},
$$

where $\bar{V}$ denotes the sum of the bare potential and all pp ladder diagrams. The hard-core limit is simply

$$
\bar{V}(p)=4 \pi a(\sin p a) / p a .
$$

As we have seen above, the quantity $\bar{V}(0)$ is frequently of importance in determining the radius of convergence of perturbative expansions. Here, $\bar{V}(0)$ is simply $4 \pi a$. This is also a good approximation to $\bar{V}(p)$ provided that $p$ is less than $1 / a$.

One can now imagine using this effective potential to sum RPA. When done correctly for Bose systems in the low-density limit, one obtains the classic result of Lee and Yang. Some care is required to obtain this result. We shall discuss this case below. Here, we consider the possibility that eq. (38) might serve as a suitable driving term for a perturbative treatment of the fermion RPA. We have seen that there is a finite radius of convergence in this case, eq. (32). For the present choice of $\bar{V}(p)$, we find that

$$
\rho<\pi / 192 a^{3}
$$

if the Fermi RPA is to converge. The point of this estimate is that there is now a natural maximum density available - the volume of the hard spheres themselves - which allows us to decide if this critical density for convergence is large or small. It is small,

$$
\rho_{\max } / \rho_{\mathrm{c}}=1152 / \pi^{2}=116.7 \text {. }
$$

This is a very serious restriction. While a perturbative treatment of the Fermi RPA may be appropriate for imperfect gases, it will almost never be appropriate for liquids. For example, the equilibrium density of nuclear matter is approximately $1 / 7$ the maximum density. We are more than an order of magnitude outside the radius of convergence. $\left.{ }^{* *}\right)$ While this seems (and is) obvious, a startling number of nuclear-matter calculations are based on a "hole line" expansion which implicitly involves a perturbative treatment of the RPA. This expansion will not converge at the equilibrium

*) If $E_{0}$ is set at $-20 \mathrm{MeV}$, the smallest eigenvalue changes slightly - and in the expected direction - to -0.071 .

**) Since nuclear matter has a degeneracy $\nu=4$, the ratio of eq. (40) must be doubled. 
density or at any other density for which a locally stable homogeneous solution exists. Making the conservative ${ }^{*)}$ assumption that $E / A$ is a quadratic function of $\rho$ with a minimum at $\rho_{0}$, one immediately concludes that the compressibility will be negative for densities less than $2 \rho_{0} / 3$. For all lower densities, a homogeneous phase will be unstable and the liquid will necessarily cavitate. Clearly, the spinodal density is also an order of magnitude outside the radius of convergence of the RPA.

Barring special dynamical circumstances, both the pp ladders and the RPA (or ph chains) must be summed to all orders to obtain a convergent description of quantum liquids. This is true of nuclear matter and, of course, of liquid helium. Unfortunately, the willingness and ability to sum both sets of diagrams is not sufficient. To see this we must look beyond the energy of the system. A sensible requirement for an acceptable many-body theory is that it provides a simultaneous description of long-range correlations and short-range correlations. As we have seen, the best monitor of long range correlations is the small- $k$ behaviour of $S(k)$. The related monitor of short-range correlations is the distribution function, $g\left(r_{12}\right)$, which is the probability that there will be a particle at $r_{2}$ given a particle at $r_{1}$. The quantity $\rho[g(r)-1]$ is simply the Fourier transform of $S(k)-1$. Thus, a reliable theory for a system with strong short-range repulsion will yield $S(k) \sim k$ as $k \rightarrow 0$ and $g(r) \rightarrow 0$ as $r \rightarrow 0$. The full RPA summation is required to create long-range correlations. The full pp ladder summation is required to create short-range correlations. All RPA processes are ph reducible and, hence, $p p$ irreducible. They represent suitable driving terms for the pp ladders. Similarly all pp ladder diagrams are ph irreducible and suitable driving terms for the chain diagrams of the RPA. Thus, one might consider an iterative scheme in which we alternately perform complete $\mathrm{pp}$ and $\mathrm{ph}$ summations with driving terms of increasing sophistication. For example, one could:

(i) Sum the pp ladders with the bare potential.

(ii) Add these pp ladders to the bare potential to form a new effective ph interaction and sum the RPA. Stopping at this step will leave us with good long-range correlation but an inadequate description of short-range correlations.

(iii) Add these ph chains to the bare potential to form a new effective pp interaction and sum the pp ladders. Stopping at this step will leave us with good short-range correlation but an inadequate description of long-range correlations.

(iv) Return to step (ii) if desired.

Unfortunately, there is no place to stop this procedure. Stopping after step (ii) will generally lead to an inadequate description of short-range correlations. Stopping after step (iii) will generally destroy the linear vanishing of $S(k)$. The only acceptable strategy is to repeat these steps infinitely many times until we achieve a self-consistent treatment of both the pp ladders and the ph chains.

Let us return to the special ${ }^{* *)}$ but important example of a Bose system with a hard-core potential where we are again prepared to "perform" pp ladder sums by the replacement of eq. (38). These ladder sums can then be used to drive the RPA leading to an energy similar to eq. (26) with the replacement $V \rightarrow \bar{V}$. There are several subtleties here associated with the correct counting of diagrams which must be handled with care. For example, the explicit energy expression given by eq. (26) must be modified. This expression contains a term of order $V^{2}$ which is identical in structure and has already been included in the pp ladder diagrams through the replacement of $V$ by $\bar{V}$. This term must be removed. There are no other counting errors at this level and, with this modification, the resulting energy is precisely the familiar result of Lee and Yang [see eq. (59)

*) Forms of $E(\rho)$ more realistic than the simple form considered here will place the spinodal density closer to the equilibrium density.

**) This problem is special in the sense that we do not have the option of stopping the above procedure at step (ii). Doing so will result in an infinite energy [see eq. (42) below]. 
below]. Note that this energy is finite and vanishes like $\rho a$ in the low-density limit.

Greater care is required to avoid counting errors in the determination of the associated static structure function. The simple replacement of $V$ by $\bar{V}$ in eq. (23) would lead to serious counting errors. Even the expectation, based on eq. (23), that $S(k)$ should vanish linearly is upset when diagrams are counted correctly. The safest course is to take the energy expression just constructed and recast it in precisely the form of the Feynman-Hellmann theorem. This is somewhat tricky since our energy expression involves the effective potential, $\bar{V}$, and the Feynman-Hellmann theorem requires the bare potential, $V$. This recasting will require judicious use of the fact that our effective potential is the two-body scattering amplitude which satisfies the equation

$$
\bar{V}=V+V G_{\mathrm{pp}} \bar{V} \text {. }
$$

Once our energy has been cast in this form, $S(k)$ follows by an appropriate differentiation with respect to the coupling constant. This is a tedious procedure. Fortunately, we do not need to carry it out explicitly provided that one accepts this definition of $S(k)$ through the Feynman-Hellmann theorem. Then, any two methods which yield the same expression for the energy (as a function of the coupling constant) will necessarily yield the same expression for $S(k)$. In the next section we will obtain the present energy using an argument specifically designed to avoid counting errors. The present procedure, if carried out, is guaranteed to yield the static structure function given in eq. (60) below. As we shall see there, the resulting structure function does not vanish linearly with $k$ in the small- $k$ limit. The associated distribution function will remain zero inside the hard-core radius. The reason for this is physically obvious and merits discussion here. Returning to eq. (25), we see that the energy of a Bose system can be rewritten in coordinate space as

$$
\frac{E}{A}=\frac{1}{2} \rho \int_{0}^{1} \mathrm{~d} \lambda \int \mathrm{d}^{3} r V(r) g_{\lambda}(r) .
$$

The hard-core potential, $V(r)$, is strictly infinite for all $r<a$. Thus, if the energy of the hard-core system in any approximation is finite (as here), the associated approximate $g(r)$ must be strictly zero inside the hard-core radius. We shall return to this point below.

The procedure suggested above is the simplest scheme which can provide a reasonable simultaneous description of both short and long wavelength correlations along with the modest confidence that the various diagrams not excluded have not already been demonstrated to diverge. (This confidence falls far short of the elusive goal of actually demonstrating the convergence of the perturbative treatment of missing diagrams.) This self-consistent summation is precisely the content of the "parquet theory" to which we now turn our attention. While one may be required to do even more, we have demonstrated step-by-step that doing less ensures the failure of a many-body theory.

\section{Parquet theory}

It is our intention here to provide only a brief description of the parquet approach to the Bose many-body problem. (A more detailed exposition can be found in ref. [22].) The basic entity in parquet theory is the four-point function, $\Gamma$, which is to be constructed as the self-consistent sum of all pp ladder diagrams and all ph chain diagrams. Accordingly, each diagram contributing to $\Gamma$ is either a bare potential, a ladder diagram $(L)$ which is pp reducible (and ph irreducible) or a chain diagram $(C)$ which is ph reducible (and pp irreducible). Hence,

$$
\Gamma=V+L+C
$$




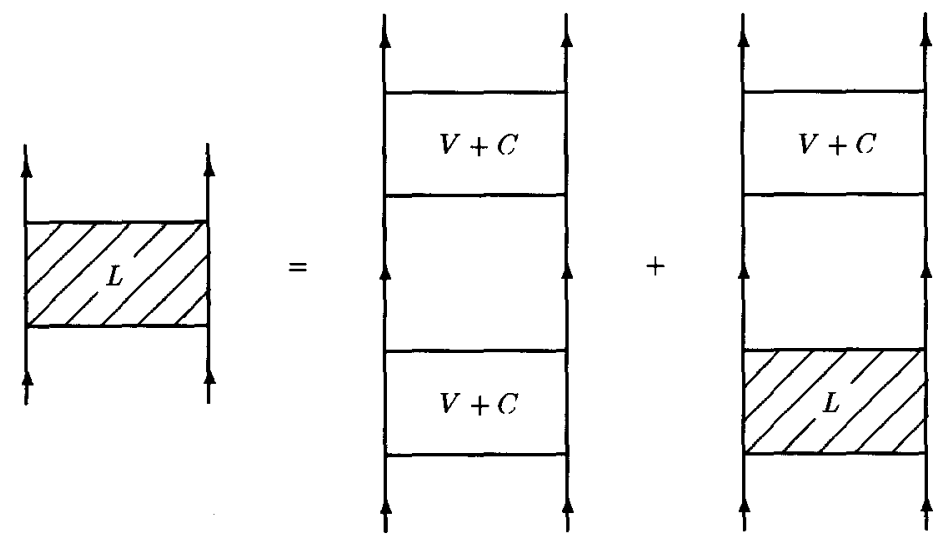

Fig. 2. Diagrammatic representation of the parquet equation for $L$.

with

$$
\begin{aligned}
& L=(V+C) G_{\mathrm{pp}}(V+C)+(V+C) G_{\mathrm{pp}} L, \\
& C=(V+L) G_{\mathrm{ph}}(V+L)+(V+L) G_{\mathrm{ph}} C .
\end{aligned}
$$

The laddering and chaining operations are schematically shown in figs. 2 and 3.

The propagators $G_{\mathrm{pp}}$ and $G_{\mathrm{ph}}$ are each the product of two bare single-particle propagators. Since we adopt a Bose formulation which is the limit of a Fermi formulation (as described above), these bare propagators are given by eq. (16). Any approximation to $\Gamma$ obtained from some restricted class of diagrams will necessarily have counting problems. ${ }^{*)}$ For parquet theory, the counting rules needed to obtain the proper self-energy, $\Sigma^{\star}$, are extremely simple: Select from the parquet $\Gamma$ those ladder diagrams whose top rung is $V$. Close one particle line with a single-particle Green's function. Discard all other diagrams. The resulting $\Sigma^{\star}$ is shown in fig. 4. Proceeding from the proper selfenergy to $E / A$ is straightforward. In general, there are counting problems in going from $\Sigma^{\star}$ to $E / A$ which are the precise analogues of those encountered in going from $\Gamma$ to $\Sigma^{\star}$. In the latter case, no approximate diagrammatic theory can avoid such difficulties. Fortunately, it is possible to construct

*) For the exact $\Gamma$ and its associated proper self-energy, $\Sigma^{*}$, the functional differentiation of an $n$th order contribution to $\Sigma^{\star}$ with respect to the single-particle Green's function will lead to $n$ diagrams contributing to $\Gamma$. Conversely, in closing off all $n$ such diagrams contributing to $\Gamma$ in order to construct $\Sigma^{\star}$, a factor of $1 / n$ is required. For any approximate theory there will be terms in $\Sigma^{\star}$ such that some (but not all) of their $n$ contributions to $\Gamma$ are missing. Thus, explicit counting rules are required in going from $\Gamma$ to $\Sigma^{\star}$ in any approximate theory.
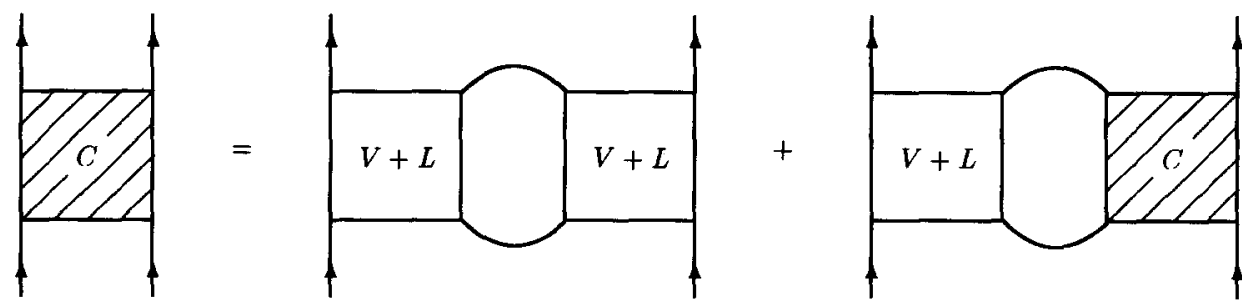

Fig. 3. Diagrammatic representation of the parquet equation for $C$. 


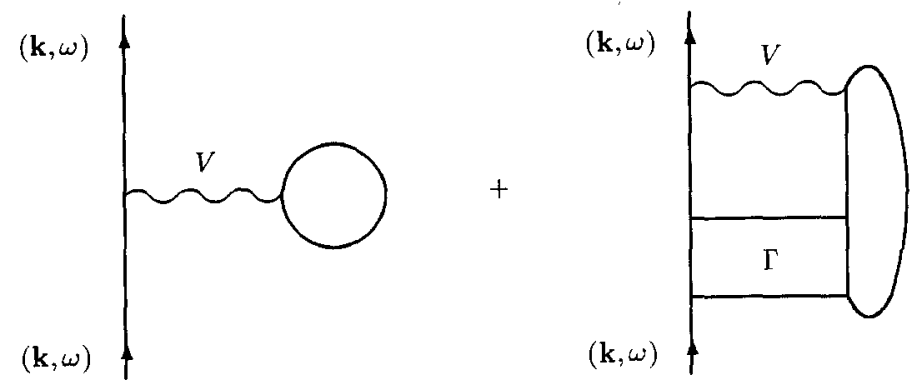

Fig. 4. The proper self-energy, $\Sigma^{\star}(k, \omega)$, in parquet theory.

approximate theories without counting difficulties in going from $\Sigma^{\star}$ to $E / A$. Even more fortunately, parquet theory is the simplest theory which is free of these complications. ${ }^{* *}$ ) As a consequence, all legitimate routes from $\Sigma^{\star}$ to $E / A$ yield the same answer in parquet theory.

Finally, the static structure function in parquet approximation can be obtained with the FeynmanHellmann theorem as

$$
S(k)=1-\left.\left(\rho / k^{2}\right)(\mathrm{d} / \mathrm{d} \alpha)\left[\alpha\left(\alpha V+L^{\alpha}+C^{\alpha}\right)\right]\right|_{\alpha=1},
$$

where $C^{\alpha}$ and $L^{\alpha}$ are obtained by solving the above equations with $V$ replaced by $\alpha V$.

In practice, the parquet equations have many variables. Thus, over and above the diagrammatic truncations of the parquet theory, it is necessary to make certain kinematic approximations aimed at reducing $L$ and $C$ to functions of the three-momentum transfer only. These approximations are most easily realized by making local approximations to the propagators $G_{\mathrm{pp}}$ and $G_{\mathrm{ph}}$. They can be constructed uniquely by the requirement that these approximate propagators reproduce the energy (and static structure functions) of the pure pp ladders and pure ph chains, respectively. This leads us to the following form for the parquet equations:

$$
\begin{aligned}
& L(k)=\int \frac{\mathrm{d}^{3} p}{(2 \pi)^{3}}[V(p+k)+C(p+k)] \frac{1}{\left(-2 \omega_{p}\right)}[V(p)+C(p)+L(p)], \\
& C(k)=[V(k)+L(k)] 2 \rho \omega_{k}\left(\bar{\omega}_{k}^{2}-\omega_{k}^{2}\right)^{-1}[V(k)+L(k)+C(k)], \\
& \bar{\omega}_{k}^{2}=\epsilon_{k}^{2}-\left(\epsilon_{k}+\omega_{k}\right)^{3} /\left(\epsilon_{k}+3 \omega_{k}\right), \\
& \epsilon_{k}^{2}=\omega_{k}^{2}+2 \rho \omega_{k}[V(k)+L(k)] .
\end{aligned}
$$

As usual, we adopt the notation

$$
\omega_{k}=\frac{1}{2} k^{2}
$$

and the convention that all energies are measured in units of $\hbar^{2} / m$. The parquet energy now assumes the explicit form

$$
\frac{E}{A}=\frac{1}{2} \rho V(0)+\frac{1}{2} \rho \int \frac{\mathrm{d}^{3} p}{(2 \pi)^{3}} V(p) \frac{1}{\left(-2 \omega_{p}\right)}[V(p)+L(p)+C(p)] .
$$

**) This is true for the following reason. If one differentiates an $n$th order contribution to $E / A$ with respect to the single-particle Green's function, one will get $n$ contributions to $\Sigma^{\star}$. If the diagram in question is a parquet contribution to $E / A$, all $n$ contributions to $\Sigma^{\star}$ are also of the parquet class. 
Table 1

Comparison of parquet, perturbatively improved parquet and Monte Carlo results for the ground-state energy of the charged Bose fluid $[23,26], r_{\mathbf{s}}$ is in units of Bohr radii, $E$ in units of rydberg.

\begin{tabular}{rlll}
\hline \multicolumn{1}{c}{$r_{\mathrm{s}}$} & $E_{\text {parquet }}$ & $E_{\text {parquet }}^{\text {pert. }}$ & $E_{\mathrm{MC}}$ \\
\hline 2 & -0.4501 & -0.4555 & $-0.4531(1)$ \\
5 & -0.2149 & -0.2185 & $-0.21663(6)$ \\
10 & -0.1202 & -0.1216 & $-0.12150(3)$ \\
20 & -0.0658 & -0.0665 & $-0.06666(2)$ \\
\hline
\end{tabular}

Table 2

Comparison of parquet, perturbatively improved parquet and GFMC results for the ground-state energy of the hardsphere gas $[23,21] . \underline{E}$ is in units of $\hbar^{2} / m a^{2}$.

\begin{tabular}{lllr}
\hline$\rho a^{3}$ & $E_{\text {parquet }}$ & $E_{\text {parquet }}^{\text {pert. }}$ & \multicolumn{1}{l}{$E_{\mathrm{GFMC}}$} \\
\hline 0.166 & 4.25 & 3.98 & $4.24(5)$ \\
0.200 & 5.90 & 5.49 & $5.80(5)$ \\
0.244 & 8.69 & 7.96 & $8.28(9)$ \\
0.270 & 10.52 & 9.74 & $10.65(7)$ \\
\hline
\end{tabular}

The static liquid structure function is defined precisely as in eq. (46) above. Given the local approximations made, the dynamic structure function is not accessible in a reliable fashion.

It is understood that perturbative improvements of parquet theory will involve the modification of the driving term, $V$, to include processes not included. ${ }^{*)}$ Again, there is no guarantee that this perturbative improvement of $V$ will result in a convergent expansion for either the energy or the structure function. But, as we have taken some pains to point out above, to do less will certainly result in one (or more) of three disasters. The perturbative improvement of this subminimal theory will not converge and/or short-range correlations will be incorrect and/or long-range correlations (and the velocity of sound) will be catastrophically wrong.

Two comments are in order before returning to the Bose problem with hard-core potentials. First, parquet theory as outlined above is well suited to numerical implementation, and its results have been compared with those of Green's function Monte Carlo calculations in a number of cases [23]. These systems include the hard-core potential (for densities close to the close packed density) [21], various potentials appropriate for the description of liquid ${ }^{4} \mathrm{He}[24,25]$ and the Bose Coulomb gas problem [26]. Some comparisons, including results from perturbatively improved parquet calculations, are given in tabs. 1 and 2 and figs. 5 and 6 . In all cases agreement between parquet and GFMC results for $E / A$ is at the "few percent" level, and agreement for $S(k)$ is within the rather substantial statistical uncertainties associated with the GFMC results. Parquet theory at least passes the "proof of the pudding". Second, we note that the local approximation to parquet theory has an appealing connection to the hypernetted chain "variational" calculations based on a Jastrow trial function $[28,29]$. The Euler equation obtained when the HNC energy is functionally minimized with respect to the two-body distribution function is identical to our local approximation to parquet theory with the addition of one well-defined subclass of diagrams [30] ${ }^{* *}$. Except for this error, energies and structure functions from such optimized HNC calculations are identical to parquet results. Thus, the optimized $\mathrm{HNC}$ is, from our point of view, an equally acceptable starting point for a reliable many-body theory. (In the case of the optimized $\mathrm{HNC}$, perturbative improvements have been studied under the name of the Method of Correlated Basis Functions.)

We now return to the hard-core boson problem (in the dilute limit) armed with the more powerful techniques of parquet theory. (Here, we shall use the parquet results to ensure proper counting for both the energy and the static structure function and to measure the importance of those terms neglected by traditional low-density truncations.) Since $V(k)$ is infinite and since this infinity is controlled by the pp ladder sums, we must express everything in terms of the finite sum of pp

\footnotetext{
*) These modifications can always be expressed in terms of the parquet $\Gamma$ which, for strong bare potentials, is the smallest quantity available.

${ }^{* *)}$ For purposes of evaluating $S(k)$ and $E / A$, the addition of these diagrams is numerically unimportant.
} 


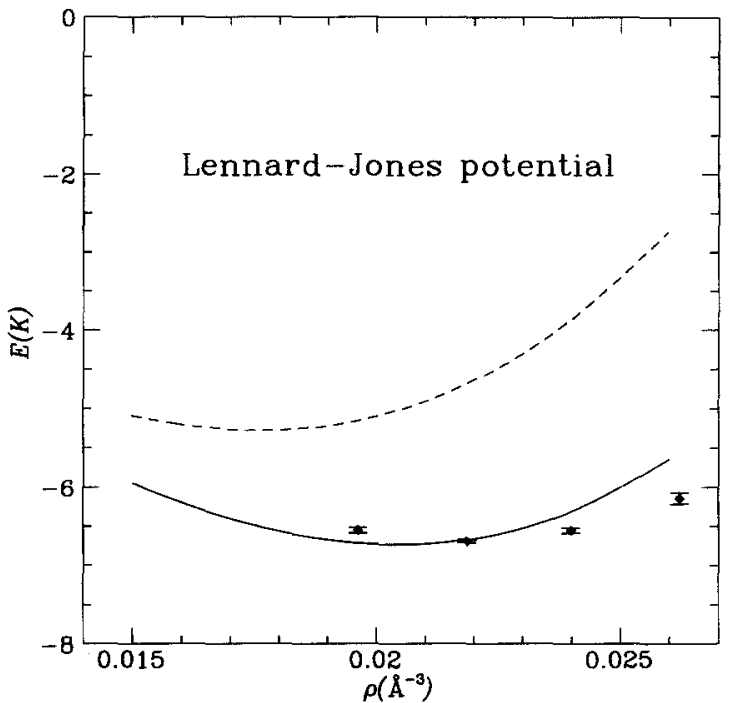

Fig. 5. Energies versus density for the Lennard-Jones potential. The dashed line represents energies obtained from the unaltered parquet equations, the solid line energies obtained from pertubatively improved parquet equations [23]. The data points are GFMC results [24].

ladders obtained from the bare potential. This quantity, which we shall denote by $V(k)+\bar{L}(k)$, is

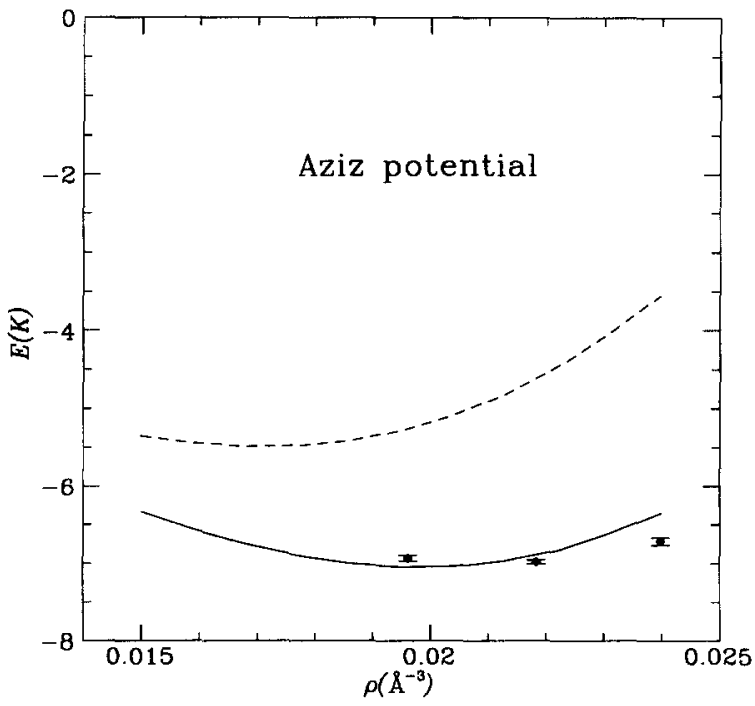

Fig. 6. Energies versus density for the HFDHE2 potential of Aziz et al. [27]. The dashed line represents energies obtained from the unaltered parquet equations, the solid line energies obtained from pertubatively improved parquet equations [23]. The data points are GFMC results [25]. 
given by eq. (38) as

$$
V(k)+\bar{L}(k)=4 \pi a(\sin k a) / k a .
$$

We expect that the difference between the correct pp ladder sums and this zero density approximation, $\delta L=L-\bar{L}$, will be small in the dilute limit. Thus, it is convenient to rewrite eq. (52) as

$$
\begin{aligned}
\frac{E}{A}= & \frac{1}{2} \rho[V(0)+L(0)]-\frac{1}{2} \rho \int \frac{\mathrm{d}^{3} p}{(2 \pi)^{3}} C(p) \frac{1}{\left(-2 \omega_{p}\right)}[V(p)+L(p)+C(p)] \\
= & \frac{1}{2} \rho[V(0)+\bar{L}(0)]-\frac{1}{2} \rho \int \frac{\mathrm{d}^{3} p}{(2 \pi)^{3}} C(p) \frac{1}{\left(-2 \omega_{p}\right)}[V(p)+\bar{L}(p)+C(p)] \\
& +\frac{1}{2} \rho \delta L(0)-\frac{1}{2} \rho \int \frac{\mathrm{d}^{3} p}{(2 \pi)^{3}} C(p) \frac{1}{\left(-2 \omega_{p}\right)} \delta L(p) .
\end{aligned}
$$

The definition of $C(k)$, eq. (48), reveals an explicit factor of the density which suggests that $C(k)$ is small. This is misleading since the definition of $\Gamma(k)$ implies that

$$
\lim _{k \rightarrow 0} \Gamma(k)=0 .
$$

This means that, in the limit $k \rightarrow 0, C(k)$ is precisely $-[V(k)+L(k)]$ independent of $\rho$. However, the range over which $C(k)$ is comparable to $V(k)+L(k)$ decreases with the density so that $C(k)$ may be considered to be genuinely small unless

$$
k<4 \sqrt{\pi \rho a} .
$$

The leading corrections to the energy will thus come from the small- $k$ contributions to various integrals, and we will be entitled to replace $V(k)+\bar{L}(k)$ by $4 \pi a$ in this region. This replacement is extremely convenient since it enables us to redefine variables in the various correction terms to $E / A$ so that all dimensioned quantities (e.g., $\rho$ and $a$ ) will appear only as prefactors. We emphasize, however, that this replacement does not entail any additional approximations in the present limit. In order to construct $\delta L$, it is necessary to know $C(k)$. For this purpose it is appropriate to construct $C(k)$ using $(V+\bar{L})$. In fact, there are no other leading-order corrections to $C(k)$. Given $C(k)$, we construct $\delta L$ using the standard "two potential" results summarized in appendix B. To leading order we obtain

$$
\delta L=(V+\bar{L}) G_{\mathrm{pp}} C+C G_{\mathrm{pp}}(V+\bar{L})+C G_{\mathrm{pp}} C .
$$

Each additional iteration of the ladder equation would introduce an additional power of $\left.\left(\rho a^{3}\right)^{1 / 2} .^{*}\right)$ We are not interested in these higher corrections.

The energy of the hard-core system is now obtained by inserting this $\delta L$ into eq. (54) above. There are a number of desirable cancellations so that the final expression for the energy becomes

$$
\frac{E}{A}=\frac{1}{2} \rho[V(0)+\bar{L}(0)]+\frac{1}{2} \rho \int \frac{\mathrm{d}^{3} p}{(2 \pi)^{3}}[V(p)+\bar{L}(p)] \frac{1}{\left(-2 \omega_{p}\right)} C(p) .
$$

*) More precisely, neglected contributions to $E / A$ will be either of-order $\rho^{2} a^{4}$ or $\rho^{2} a^{4} \ln \rho a^{3}$. 
Given the definition of $C(p)$ above, it is clear that the leading behaviour of the second term here can be obtained with $V(p)+\bar{L}(p)$ replaced by $4 \pi a$ as discussed above. This integral is easily done and yields

$$
E / A=2 \pi \rho a\left[1+(128 / 15)\left(\rho a^{3} / \pi\right)^{1 / 2}\right] .
$$

This expression is precisely the Lee-Yang result [20]. As noted in the last section, this result can also be obtained by slight counting modifications of the RPA. Here, the counting is correct by construction and the fact that additional diagrams are of higher order is made more transparent.

Let us now consider the static structure function for this problem which results from the values of $L$ and $C$ just obtained. Returning to the definition, eq. (46), we see that the special properties of the hard-core potential render $V+\bar{L}$ independent of the "strength" of the potential. In this case $S(k)$ is simply

$$
\begin{aligned}
S(k)= & 1-\left(\rho / k^{2}\right)[V(k)+\bar{L}(k)+\delta L(k)+C(k)] \\
= & 1-\left(\rho / k^{2}\right)[V+\bar{L}+C]-\left(\rho / k^{2}\right)\left[(V+\bar{L}) G_{\mathrm{pp}} C\right] \\
& -\left(\rho / k^{2}\right)\left[C G_{\mathrm{pp}}(V+\bar{L}+C)\right] .
\end{aligned}
$$

Again, $C$ is driven by $V+\bar{L}$. The second of these forms is most useful. The final term there is of higher order in $\left(\rho a^{3}\right)^{1 / 2}$ due to the cancellations inherent in $\Gamma(k)$ as discussed above. We will discard it. The sum of the first two terms is

$$
2 \omega_{k} /\left(\epsilon_{k}+\omega_{k}\right)
$$

where $\epsilon_{k}$ is obtained from eq. (50) with the replacement $V \rightarrow V+\bar{L}$. These two terms vanish linearly in the small- $k$ limit as one would expect from the RPA. Unfortunately, the remaining (third) term does not vanish linearly as $k \rightarrow 0$.

It is easy to evaluate the distribution function at $r=0, g(0)$, given this form for the static structure function. We simply integrate $[S(k)-1] / \rho$ over $\mathrm{d}^{3} k$ and add 1 . The final term can again be neglected. The sum of the first two terms yields

$$
(128 / 15) \sqrt{\rho a^{3} / \pi} \text {. }
$$

The third term precisely cancels this term leaving us with a result which is 0 to the order considered. (Corrections terms are of order $\rho a^{3}$.) The third term in eq. (60) is evidently the most interesting term. Its inclusion spoils the small- $k$ behaviour of $S(k)$ and corrects the small-r behaviour of $g(r)$. This emphasizes the difficulties inherent in truncating the parquet scheme short of self-consistency. The inclusion of this term (or similar terms in higher orders) is required if we are to obtain a finite energy. This is consistent with our discussion in the last section.

The primary purpose of this demonstration was not to obtain a famous result with considerable economy although that is an interesting byproduct. At the level to which we have worked, parquet theory does not miss any diagrams. Further, it evaluates all diagrams required at this order correctly by design. Success was guaranteed. This derivation does offer a way of thinking about the Lee-Yang result which may appeal to devotees of diagrams. We find it satisfying that this result emerges so naturally from the simplest many-body theory which is not demonstrably pathological! The primary observation here is that terms which improve the description of $g(r)$ for small $r$ degrade the description of $S(k)$ for small $k$ and vice versa. The order of this degradation [in powers of 
$\left(\rho a^{3}\right)^{1 / 2}$ ] can be raised by undertaking additional iterations of the parquet equations. It cannot be eliminated entirely - as it should be in the present example - unless the parquet equations are solved to full self-consistency. Finally, the nature of the hard-core potential and the desire for a finite energy force this error to be in $S(k)$ and not in $g(r)$.

We note that reproducing the Lee-Yang result at this level does not provide a test of the validity of either the diagrammatic truncations of parquet theory or of the kinematic approximations adopted. Such a test would require the evaluation of the next terms contributing to the energy. A portion of this correction has been obtained by $\mathrm{Wu}$ who found [31]

$$
\delta E / A=16 \pi \rho a(4 \pi / 3-\sqrt{3}) \rho a^{3} \ln \rho a^{3}+\mathrm{O}\left(\rho^{2} a^{4}\right) .
$$

Although complicated, the complete evaluation of this term is perfectly feasible within the parquet scheme. Even as it stands, eq. (61) allows us to gauge the rough range of validity of eq. (59). If we require, for example, that this $\delta E$ is $10 \%$ of the second term in eq. (59), we find that $\rho a^{3}$ should be less than $4 \times 10^{-6}$. This is to be compared with the maximum value of $\rho a^{3}$ which is $6 / \pi$. Note also that GFMC calculations have only been performed in the vicinity of this maximum density and, for technical reasons, are not readily extended into the range of applicability of the Lee-Yang results. Given the success of parquet theory in these two distant limits, one is encouraged to take seriously parquet results in the intermediate-density regime where no other results are available.

\section{Failures of parquet theory}

Although the title of this section is negative, its conclusions are not. Any approximate theory can fail to provide reliable answers when the true dynamical solution has features not embodied in it. Linear theories usually continue to return nonsensical (or internally inconsistent) results. For example, a hole line expansion of Brueckner theory will continue to provide homogeneous solutions at all densities including those low densities where it indicates a negative compressibility and where homogeneous solutions cannot exist. The failure of nonlinear theories (like the parquet theory) is usually indicated by a failure to find solutions. The question is whether these failures actually indicate either pathological dynamics or a phase change of the system beyond the realm of applicability of the approximate theory. In the latter case, does the nature of the failure indicate the nature of the new phase? For parquet theory the answer is an emphatic yes.

Given the close connection between parquet and HNC approaches, it is useful to use HNC arguments to understand the limitations of parquet. Specifically, HNC calculations are based on a Jastrow wave function (a symmetrized product of two-body correlation functions) which has the form

$$
\Psi=\prod_{i<j} f\left(r_{i j}\right)
$$

We note that (i) there is no product of single-particle functions as in eq. (1) and (ii) all two-body correlations are identical. (In fact, it would be more accurate to say that there is a trivial product of single-particle wave functions; the individual wave functions are simply the $k=0$ plane waves in a box equal to the size of the system.) This has serious consequences. If we work in a region where the compressibility is negative, the system would like to make a macroscopic change in its density (i.e., change the size of the box). This could be accomplished with ease if single-particle wave functions were present in eq. (62). They are not. Instead, HNC and parquet simply fail to find 
solutions. Similarly, solidification is also most easily expressed in terms of a symmetrized product of localized single-particle wave functions. Again, this possibility is excluded by the Jastrow form. Again, HNC and parquet simply fail to find solutions. The final failure occurs when the effective pp interaction in the medium $-V+C$ in the parquet language - is sufficiently strong to form two-body bound states (molecules) in the system. This would require an extension of the Jastrow form to have two very different kinds of correlation functions. Instead, HNC and parquet fail when the system actually wishes to undergo a transition from an atomic to a molecular phase.

The reflection of these instabilities is quite clear in parquet theory. First, we note that the Calogero pathology is manifest. Consider the case where $V(k)$ is attractive for all $k$. It is clear from the definition in eq. (48) that $C(k)<0$ for all $k$. Every term in a perturbative solution of eq. (47) is also negative suggesting that $L(k)<0$ for all $k$. In particular, $V(0)+L(0)$ will be less than zero. This indicates that, as $k \rightarrow 0$, we will encounter a square-root singularity (in $\epsilon_{k}$ ) in eq. (50) and the parquet equations will fail. This is precisely the singularity noted in our earlier discussions of the RPA. It can also happen that $V(0)+L(0)<0$ at subequilibrium densities even when the bare potential satisfies Calogero's conditions. *) As $V(0)+L(0) \rightarrow 0$ (from above), the compressibility also approaches zero as does the coefficient of the linear term in $S(k)$. With the guidance of Bijl-Feynman, this is to be viewed as a lowering of the energy of the system through the creation of a phonon of a wave length comparable to the box size. Phonons will condense in this state producing a macroscopic change in the density of the system - cavitation.

This observation allows us to be more specific about the necessity of summing the RPA in Fermi systems. As we have seen, there is a finite (but often small) radius of convergence. Now we can make a more physical statement about this radius of convergence. All stable, self-bound (Fermi) systems have a spinodal density below which the compressibility is negative and the system is unstable with respect to small, long wave length fluctuations. This instability is always indicated by the diseases of the Fermi RPA. Specifically, the RPA cannot converge in this region. Thus, one is never entitled to treat the RPA perturbatively for any self-bound Fermi system. Since we usually know in advance where the dynamical system in question is expected to be bound, we can usually make a reliable a priori determination of the convergence of the Fermi RPA.

Similarly, it is possible (for strongly repulsive interactions) that $V(k)+L(k)$ will be negative over some range in $k$. Indeed, it can be sufficiently negative that $\epsilon_{k}$ will vanish at some $k_{0} \neq 0$. Any attempt to go to higher densities will encounter square-root singularities. When this occurs, $S\left(k_{0}\right)$ becomes infinite. But infinities in $S(k)$ are the rule in the scattering from crystals. They are simply Bragg peaks which occur whenever $k$ is a reciprocal lattice vector. In parquet language, we see that we lower the energy of the system by creating phonons of wave number $k_{0}$. This condensate of phonons produces macroscopic density fluctuations also characterized by this wave number. A crystal - now regarded as a condensate of phonons with finite wave number - results. This view is supported by both parquet and HNC calculations. Both reveal that the (finite) peaks in $S(k)$ grow in height as the density increases and that they occur at values of $k$ which approach the values given by the reciprocal lattice vectors (at the same density). Since optimized HNC calculations arise from the functional minimization of an approximate energy functional, one can also study the spectrum of small amplitude fluctuations through the related second-order variational problem. The resulting spectrum (in HNC approximation) can be categorized by both a wave number and an angular momentum, $\left.l .{ }^{* *}\right) \mathrm{HNC}$ results for the Lennard-Jones potential and hard-core systems show that the soft modes have $l$ equal to 5,6 and 7 . These are the crystal harmonics required to

*) When the liquid is self-bound, this always occurs at sufficiently low densities.

**) More correctly, $l$ labels a crystal harmonic. 
build either fcc or hcp close packed lattices. In the case of the Bose Coulomb gas, the soft mode has $l$ equal to 4 which indicates an instability towards the formation of a bcc lattice. In each case, the nature of the unstable mode in HNC correctly identifies the crystal symmetry of the solid phase.

We have actually had some preparation for this result. For sufficiently "hard" potentials, the Fourier transform, $V(k)$, is not always positive even though $V(r)>0$ for all $r$. As we have seen in connection with eq. (37), the Fourier transform of a square well is

$$
V(k)=\left[4 \pi a^{3} V_{0} /(k a)^{2}\right][(\sin k a) / k a-\cos k a] .
$$

For sufficiently high densities, we will always encounter the square-root problem at some finite $k$. This is not a disease of the RPA. Rather, it is a signature of incipient solidification.

We can also study parquet by increasing the strength of $V$. For a potential which has both short-range repulsion and longer-range attraction, this will eventually result in a bound state at zero energy. Thus, for some critical strength, $V+L$ will develop a pole. At this pole, $V(k)+L(k)$ will become infinite for all $k$. As the strength of $V$ is further increased and we move through this pole, the sign of $V(k)+L(k)$ will change. We will again encounter square-root singularities. This is exactly what happens in the full parquet equations. In practice, the presence of the chains in eq. (47) delays this instability but does not prevent it.

Each of these three failures is sensible, and each has its signature. The vanishing of $V(0)+L(0)$ indicates the spinodal. The vanishing of $\epsilon_{k}$ at $k \neq 0$ indicates solidification. A pole in $\Gamma(k)$ for all $k$ indicates the formation of a two-body bound state.

A similar situation arises in the artificial extension of the parquet equations to systems of "spin 1/2-isospin $1 / 2$ bosons" interacting with the full tensor structure associated with the NN interaction. A richer structure of interpretable instabilities (including pion condensation) emerges [32].

Even the failures of parquet theory must be counted among its successes.

\section{Summary and conclusions}

We have moved slowly and with simple arguments from Calogero's elementary observation towards a minimal acceptable version of many-body theory. The desire to reflect Calogero's instabilities and the related desire to have a many-body theory which admits convergent perturbative improvement literally forced us to sum the ph diagrams of the RPA for bosons (at any density and for any interaction) and for fermions (for most interactions and densities of interest). ${ }^{*}$ ) We have seen that, for virtually all realistic interactions, it is also necessary to sum the pp ladders. Further, a simultaneous description of both short-range correlations and long-range correlations requires the self-consistent summation of ph and pp ladder diagrams. The simplest theory which meets all of these criteria is the parquet theory. We have not demonstrated that parquet theory is free of difficulties. Rather, we have shown that it is the simplest theory which is not obviously diseased.

Fortunately, parquet theory seems to work in practice. The remarkable agreement between parquet results and both analytic results and GFMC results over six orders of magnitude in the density for the hard-core boson problem is encouraging.

The most important unsolved problem at zero temperature is, in our opinion, the extension of parquet theory to Fermi systems. The problem is formally complicated by the fact that, in Fermi systems, there are two distinct particle-hole channels. Correspondingly, the parquet equations for

*) Certainly, the RPA is a necessity for all self-bound Fermi systems. 
$\Gamma$, eqs. (44) and (45), must be supplemented by a third equation. Such formal work has already been done [33]. The remaining problem is to find reliable kinematic approximations which permit the reduction of the Fermi parquet equations to functions of a single variable, the magnitude of the three-momentum transfer. This task is likely to be challenging since even the pure Fermi RPA required approximations to reduce it to the desired simple form. Given the formal justification for parquet and the numerical success of its Bose counterpart, this endeavor would seem very much worth while.

We close with one general observation. Many-body theory has a well deserved reputation for being formal and complicated. Through the years, a large number of powerful techniques have been devised to enumerate diagrams and to evaluate them. Rather less attention has been given to the development of strategies for the use of this arsenal. Our goal here has been to suggest the simplest strategy which is not guaranteed to fail. Very simple arguments lead us to the necessity of a rather large class of diagrams. It is entirely possible that richer arguments might demonstrate the inadequacy of this class and force its extension. In any event, a greater concern with the strategy of the many-body problem is highly desirable.

\section{Appendix A. Approximate fermion RPA}

Consider the dynamic structure function for fermions in RPA. One is called upon to perform the summation

$$
\begin{aligned}
& S(k, \omega)=(\mathrm{i} / \rho) \Pi^{0}(k, \omega)+\Pi^{0}(k, \omega) V(k) S(k, \omega), \\
& \Pi^{0}(k, \omega)=\int \frac{\mathrm{d}^{3} p}{(2 \pi)^{3}} \theta\left(|p+k|-k_{\mathrm{F}}\right) \theta\left(k_{\mathrm{F}}-p\right) \frac{2 \omega_{p k}}{\left(\omega-\omega_{p k}+\mathrm{i} \eta\right)\left(\omega+\omega_{p k}-\mathrm{i} \eta\right)}, \\
& \omega_{p k}=\omega_{p+k}-\omega_{p}=\frac{1}{2} k^{2}+\boldsymbol{p} \cdot \boldsymbol{k} .
\end{aligned}
$$

Evidently, eq. (A.1) is simply a geometric series which is readily summed to yield

$$
S(k, \omega)=(\mathrm{i} / \rho) \Pi^{0}(k, \omega) /\left[1-\Pi^{0}(k, \omega) V(k)\right] .
$$

The difficulty is that, due to $k_{\mathrm{F}} \neq 0$, the denominator of eq. (A.2) has a nontrivial dependence on the integration variable, $p$. (This was not the case for bosons.) One can, none the less, construct $\Pi^{0}(k, \omega)$ analytically (see ref. [17]).

The result is most easily expressed in terms of the variables $q=k / k_{\mathrm{F}}$ and $\nu=m \omega / \hbar k_{\mathrm{F}}^{2}$. The real part of $\Pi^{0}(k, \omega)$ is

$$
\begin{aligned}
\operatorname{Re} \Pi^{0}(k, \omega)= & \frac{2 m k_{\mathrm{F}}}{\hbar^{2}} \frac{1}{4 \pi^{2}}\left(-1+\frac{1}{2 q}\left[1-\left(\nu / q-\frac{1}{2} q\right)^{2}\right] \ln \left|\frac{1+\left(\nu / q-\frac{1}{2} q\right)}{1-\left(\nu / q-\frac{1}{2} q\right)}\right|\right. \\
& \left.-\frac{1}{2 q}\left[1-\left(\nu / q+\frac{1}{2} q\right)^{2}\right] \ln \left|\frac{1+\left(\nu / q+\frac{1}{2} q\right)}{\mp\left(\nu / q+\frac{1}{2} q\right)}\right|\right) .
\end{aligned}
$$

The imaginary part of $\Pi^{0}(k, \omega)$ is zero except in the following regions. For $q>2$ and $q^{2} / 2+q \geq$ $\nu \geq q^{2} / 2-q$ or for $q<2$ and $q+q^{2} / 2 \geq \nu \geq q-q^{2} / 2$,

$$
\operatorname{Im} \Pi^{0}(k, \omega)=-\left(m k_{\mathrm{F}} / \hbar^{2}\right)(1 / 4 \pi q)\left[1-\left(\nu / q-\frac{1}{2} q\right)^{2}\right] \text {. }
$$


When $q<2$ and $0 \leq \nu \leq q-q^{2} / 2$,

$$
\operatorname{Im} \Pi^{0}(k, \omega)=-\left(m k_{\mathrm{F}} / \hbar^{2}\right)(1 / 4 \pi q) 2 \nu .
$$

However, in proceeding from the dynamic structure function to the static structure function, it is necessary to integrate over $\omega$. This integral is not easily done in closed form for the exact $\Pi^{0}(k, \omega)$. In proceeding further to $E / A$ it is necessary to perform a second integration over $k$. If we retain the exact form of $\Pi^{0}(k, \omega)$, we are confronted with a two-dimensional integral which must be done numerically. While this integral does not present technical difficulties, it does obscure what is actually going on. Thus, we seek an approximate evaluation of $\Pi^{0}(k, \omega)$ which leads to simpler closed forms for $S(k)$.

The $\omega$ integration is readily performed first in the case of a single factor of $\Pi^{0}(k, \omega)$ to yield

$$
S_{0}(k)= \begin{cases}\frac{3}{4} k / k_{\mathrm{F}}-\frac{1}{16}\left(k / k_{\mathrm{F}}\right)^{3} & \text { if } k \leq 2 k_{\mathrm{F}}, \\ 1 & \text { if } k>2 k_{\mathrm{F}} .\end{cases}
$$

We note immediately that the non-interacting static structure function for fermions vanishes linearly as $k \rightarrow 0$ while the free Bose structure function is always 1 .

We can find a simple (and remarkably reliable) approximation to the various higher powers of $\Pi^{0}(k, \omega)$ and, hence, the structure function by replacing $\omega_{p k}$ by some suitable $\bar{\omega}_{k}$ which is independent of $p$ in eq. (A.2). With any such $\bar{\omega}_{k}$ we can immediately integrate eq. (A.4) exactly to obtain $S(k)$,

$$
S(k)=S_{0}(k)\left[1+2 \rho S_{0}(k) V(k) / \bar{\omega}_{k}\right]^{-1 / 2} .
$$

The only question is how to determine $\bar{\omega}_{k}$.

The evident natural definition is

$$
\bar{\omega}_{k}=\frac{\int \mathrm{d}^{3} p \theta\left(|p+k|-k_{\mathrm{F}}\right) \theta\left(k_{\mathrm{F}}-p\right) \omega_{p k}}{\int \mathrm{d}^{3} p \theta\left(|p+k|-k_{\mathrm{F}}\right) \theta\left(k_{\mathrm{F}}-p\right)} .
$$

This yields

$$
\bar{\omega}_{k}=k^{2} / 2 S_{0}(k) .
$$

This average gives the sensible result that the average energy is simply $k^{2} / 2$ for $k \geq 2 k_{\mathrm{F}}$. The value $2 k k_{\mathrm{F}} / 3$ as $k \rightarrow 0$ is less immediate. With this average energy, we are led to the elementary approximation used in the text for the fermion structure function in RPA:

$$
S(k)=S_{0}(k)\left[1+4 \rho S_{0}^{2}(k) V(k) / k^{2}\right]^{-1 / 2} .
$$

\section{Appendix B. Some two-potential results}

In the process of dealing with the approximate solution to the parquet equations with a hard core interaction, it is useful to exploit some simple results regarding the pp ladder sums associated with the sum of two potentials [34]. We seek the solution to the equation

$$
T=\left(V_{1}+V_{2}\right)+\left(V_{1}+V_{2}\right) G T,
$$


under the assumptions that $V_{1}$ is too strong to admit any perturbative solution and that $V_{2}$ is sufficiently weak to admit a perturbative treatment. To this end we write this equation as two equations

$$
T_{1}=V_{1}+V_{1} G\left(T_{1}+T_{2}\right), \quad T_{2}=V_{2}+V_{2} G\left(T_{1}+T_{2}\right),
$$

with the evident definition

$$
T=T_{1}+T_{2}
$$

A symmetric rearrangement of eqs. (B.2) immediately yields

$$
T_{1}=\left(1-V_{1} G\right)^{-1} V_{1}\left(1+G T_{2}\right), \quad T_{2}=\left(1-V_{2} G\right)^{-1} V_{2}\left(1+G T_{1}\right),
$$

where the initial factor in each equation is recognized as the formal solution to the ladder equation for $V_{1}\left(\right.$ or $\left.V_{2}\right)$ alone,

$$
t_{1}=V_{1}+V_{1} G t_{1} \rightarrow t_{1}=\left(1-V_{1} G\right)^{-1} V_{1}, \quad t_{2}=V_{2}+V_{2} G t_{2} \rightarrow t_{2}=\left(1-V_{2} G\right)^{-1} V_{2} .
$$

In the present case, the sum $t_{1}$ is absolutely required for our strong potential, $V_{1}$. Thus, eqs. (B.4) become

$$
T_{1}=t_{1}+t_{1} G T_{2}, \quad T_{2}=t_{2}+t_{2} G T_{1}
$$

These equations are well suited for an iterative solution. Under the assumption that $V_{2}$ is genuinely small, for example, one finds

$$
T=t_{1}+\left(1+G t_{1}\right)^{\dagger} V_{2}\left(1+G t_{1}\right)+\mathrm{O}\left(V_{2}^{2}\right) \text {. }
$$

The use of the adjoint operator is not necessary here, but it does underscore the physical content of this result. The operator $1+G t_{1}$ is precisely the wave operator for $V_{1}$ which converts pure plane wave states into states which are dressed with the full set of correlations due to potential $V_{1}$. The second term in eq. (B.7) thus represents the usual elementary perturbation theory result in this new correlated basis.

For our purposes, we need a slightly different result which comes from the assumption that, except for the ladders of $V_{1}$ summarized in $t_{1}$, the propagator, $G$, is sufficiently small to be treated perturbatively. Thus,

$$
T=t_{1}+t_{2}+t_{1} G t_{2}+t_{2} G t_{1}+\cdots,
$$

which gives the desired result,

$$
T=t_{1}+V_{2}+V_{2} G V_{2}+t_{1} G V_{2}+V_{2} G t_{1}+\cdots \text {. }
$$

Equation (57) now follows immediately.

\section{Acknowledgements}

The authors would like to thank A. Lande for his continuing interest and advice. One of us (ADJ) would like to thank C.N. Yang for a particularly illuminating discussion of some of the topics addressed here. 


\section{References}

[1] F. Calogero, Yu.A. Simonow and E.L. Surkov, Phys. Rev. C 5 (1972) 478 .

[2] W.L. McMillan, Phys. Rev. 138 (1965) A442.

[3] E. Feenberg, Theory of quantum fluids (Academic Press, New York, 1969) p.127.

[4] A.D. Jackson, A. Lande and L.J. Lantto, Nucl. Phys. A 317 (1979) 70.

[5] F.J. Dyson, Phys. Rev, 85 (1952) 631.

[6] C.M. Bender and T.T. Wu, Phys. Rev. D 7 (1973) 1620; Phys. Rev. Lett. 37 (1976) 117.

[7] L.N. Lipatov, JETP Lett. 24 (1976) 157; JETP Lett. 25 (1977) 104.

[8] G. Parisi, Phys. Lett. 66 B (1977) 167, 382.

[9] R.J. Rivers, Path integral methods in quantum field theory (Cambridge Univ. Press, Cambridge, 1987 ) p.187.

[10] F. Bloch and A. Nordsieck, Phys. Rev. 52 (1937) 54.

[11] T. Kinoshita, B. Nižić and Y. Okamoto, Phys. Rev. Lett. 52 (1984) 717.

[12] E.H. Lieb and F.Y. Wu, Phys. Rev. Lett. 20 (1968) 1445.

[13] E.N. Economou and P.N. Poulopoulos, Phys. Rev. B 20 (1979) 4756.

[14] M. Takahashi, Prog. Theor. Phys. 44 (1970) 348; 45 (1971) 756.

[15] A. Bijl, Physica 7 (1940) 869.

[16] R.P. Feynman, Phys. Rev. 94 (1954) 262.

[17] A.L. Fetter and J.D. Walecka, Quantum Theory of Many-Particle Systems (McGraw-Hill, New York, 1971).

[18] G.E. Brown, Many-Body Problems (North-Holland, Amsterdam, 1972).

[19] K. Huang and C.N. Yang, Phys. Rev. 105 (1957) 767.

[20] T.D. Lee and C.N. Yang, Phys. Rev. 105 (1957) 1119.

[21] M.H. Kalos, D. Levesque and L. Verlet, Phys. Rev. A 9 (1974) 2178.

[22] A.D. Jackson, A. Lande and R. Smith, Phys. Rep. 86 (1982) 55.

[23] A.D. Jackson, A. Lande, R.W. Guitink and R. Smith, Phys. Rev. B 31 (1985) 403.

[24] P.A. Whitlock, D.M. Ceperley, G.V. Chester and M.H. Kalos, Phys. Rev. B 19 (1979) 5598.

[25] M.H. Kalos, M.A. Lee, P.A. Whitlock and G.V. Chester, Phys. Rev. B 24 (1981) 115.

[26] D.M. Ceperley and B.J. Alder, Phys. Rev. Lett. 45 (1980) 566.

[27] R.A. Aziz, V.P.S. Nain, J.S. Carley, W.L. Taylor and G.T. McConville, J. Chem. Phys. 70 (1979) 4330.

[28] J.M.J. van Leeuwen, J. Groeneveld and J. de Boer, Physica 25 (1959) 792.

[29] T. Morita, Prog. Theor. Phys. 20 (1958) 920.

[30] A.D. Jackson, A. Lande and R. Smith, Phys. Rev. Lett. 54 (1985) 1469.

[31] T.T. Wu, Phys. Rev. 115 (1959) 1390.

[32] A.D. Jackson and R. Smith, Nucl. Phys. A 476 (1988) 448.

[33] A. Lande and R.A. Smith, Phys. Rev. A 45 (1990) 913.

[34] A.D. Jackson and A. Lande, Nucl. Phys. A 191 (1972) 177. 